\title{
SLIP MOTION AND STABILITY OF A SINGLE DEGREE OF FREEDOM ELASTIC SYSTEM WITH RATE AND STATE DEPENDENT FRICTION
}

\author{
Ji-Cheng Gu† \\ Institute of Geophysics, \\ State Seismological Bureau, Beijing, People's Republic of China \\ JAMES R. RICE \\ Division of Applied Sciences, \\ Harvard University, Cambridge, MA 02138
}

ANDY L. RUINA

Department of Theoretical and Applied Mechanics, Cornell University, Ithaca, NY 14853

and

SIMON T. TSE

Division of Applied Sciences, Harvard University, Cambridge, MA 02138

(Received 6 April 1983)

\begin{abstract}
ABSTRACI
WE CONSIDER quasistatic motion and stability of a single degree of freedom elastic system undergoing frictional slip. The system is represented by a block (slider) slipping at speed $V$ and connected by a spring of stiffness $k$ to a point at which motion is enforced at speed $V_{0}$. We adopt rate and state dependent frictional constitutive relations for the slider which describe approximately experimental results of Dieterich and Ruina over a range of slip speeds $V$. In the simplest relation the friction stress depends additively on a term $A \ln V$ and a statc variablc $\theta$; the statc variable $\theta$ evolves, with a characteristic slip distance, to the value $-B \ln V$, where the constants $A, B$ are assumed to satisfy $B>A>0$. Limited results are presented based on a similar friction law using two state variables.

Linearized stability analysis predicts constant slip rate motion at $V_{0}$ to change from stable to unstable with a decrease in the spring stiffness $k$ below a critical value $k_{\mathrm{cr}}$. At neutral stability oscillations in slip rate are predicted. A nonlinear analysis of slip motions given here uses the Hopf bifurcation technique, direct determination of phase plane trajectories, Liapunov methods and numerical integration of the equations of motion. Small but finite amplitude limit cycles exist for one value of $k$, if one state variable is used. With two state variables oscillations exist for a small range of $k$ which undergo period doubling and then lead to apparently chaotic motions as $k$ is decreased.

Perturbations from steady sliding are imposed by step changes in the imposed load point motion. Three cases are considered : (1) the load point speed $V_{0}$ is suddenly increased; (2) the load point is stopped for some time and then moved again at a constant rate; and (3) the load point displacement suddenly jumps and then stops. In all cases, for all values of $k$, sufficiently large perturbations lead to instability. Primary conclusions are: (1) "stick-slip" instability is possible in systems for which steady sliding is stable. and (2) physical manifestation of quasistatic oscillations is sensitive to material properties, stiffness, and the nature and magnitude of load perturbations.
\end{abstract}

Wisiting, Division of Applied Sciences, Harvard University, 1981-3. 


\section{INTRODUCTION}

STICK-SLIP motions are the result of interaction of an elastic system with a frictionally slipping surface. Qualitatively, they may be related to earthquake instabilities where an elastic crust interacts with a slipping fault (BRACE and BYERLEE, 1966). Traditionally this interaction is modelled with a friction law in which the friction force is dependent on slip rate. The simplest such rate dependence is a discontinuous jump in the coefficient of friction from a 'static' value when no slip occurs down to a reduced 'dynamic' or 'kinetic' coefficient of friction when the slip rate is non-zero. Another rate dependence that leads to frictional instabilities is one for which the friction coefficient is a smooth and decreasing function of slip rate. In yet another simple friction law, the friction stress decreases with slip displacement, in a 'slip-weakening' sense, after slip begins following stationary contact (RABINOwICZ, 1958, 1959; BYERLEE, 1970). Over the years attempts have been made to associate these two kinds of friction laws (JENKIN and EWING, 1877; RABINOWICZ, 1958, 1959; DIETERICH, 1978, 1979a) and to include combinations of them in stability analysis of frictional sliding.

A more comprehensive friction law has been proposed by DiETERICH $(1978,1979 \mathrm{a})$ based on his experiments with rocks and is similar to a proposal of RABINOWICZ (1958) based on experiments with metals (SAMPSON et al., 1943). The original motivation of DIETERICH $(1978,1979 \mathrm{a})$ and RABINOWICZ $(1958)$ in proposing these laws was to unite the observed dependence of 'static' friction stress on time of nominally (see RUINA, 1983) stationary contact with the inverse dependence of steady sliding friction stress on slip rate. This unification utilized a characteristic slip distance (which is a property of the slip surface). The resulting friction laws may be said to be of a rate and state dependent type; they can manifest behavior which, depending on the modeled friction experiment, appears as any of the simpler types of friction mentioned earlier (DIETERICH, 1978, 1979a; Ruina, 1980, 1983). Recent experiments of Ruina (1980), Teufel (1981), JoHNSON (1981) and HigGs (1981) lend support for these rate and state dependent friction laws. They are expressed in Ruina $(1980,1983)$ by relations of the following form at constant normal stress $\sigma$ :

$$
\left.\begin{array}{rl}
\tau & =F\left[V, \theta_{1}, \theta_{2}, \ldots\right] \\
\dot{\theta}_{1} & =G_{1}\left[V, \theta_{1}, \theta_{2}, \ldots\right] \\
\dot{\theta}_{2} & =G_{2}\left[V, \theta_{1}, \theta_{2}, \ldots\right]
\end{array}\right\}
$$

where the dependence of $\tau$ (the shear stress transmitted across the frictional surface) on $V$ (the slip rate) and $\theta_{1}, \theta_{2}, \ldots$ is expressed by the function $F$. The state variables $\theta_{i}$, which describe phenomenologically features of the slipping surface, evolve as governed by the functions $G_{i}$ with ongoing slip (and possibly with time, depending on the details of $G_{i}$, when there is no slip). The functions $G_{i}$ are assumed to be such that the state variables evolve towards 'steady stale' values $\theta_{i}^{s s}(V)$, salisfying $G_{j}=0$ for $j=1,2, \ldots$, for slip at constant rate $V$. The corresponding steady state stress is

$$
\tau^{\mathrm{ss}}(V)-F\left[V, \theta_{1}^{\mathrm{ss}}(V), \theta_{2}^{\mathrm{ss}}(V), \ldots\right] .
$$




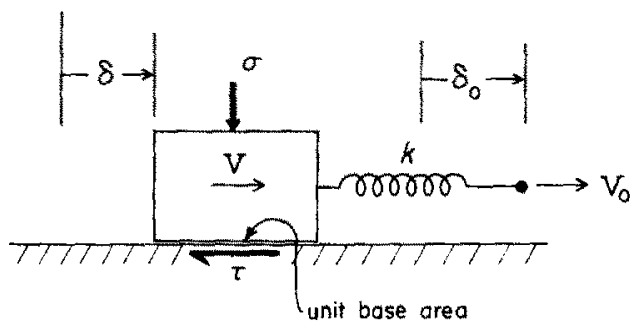

FiG. 1. Single degree of freedom elastic system. A slider of unit base area moves distance $\delta$ at the possibly varying rate $V$; the load point moves distance $\delta_{0}$ at the imposed rate $V_{0}$, stressing the slider by means of a spring with stiffess $k$. The slider mass is neglected in our analysis.

In different experiments $\mathrm{d} \tau^{\mathrm{ss}}(V) / \mathrm{d} V$ may be either positive or negative, but experiments as performed thus far suggest that $\partial F\left(V, \theta_{1}, \theta_{2}, \ldots\right) / \partial V$ is always positive. The above class of laws is mentioned or discussed in DIETFrich $(1978,1979 \mathrm{a}, \mathrm{b}, 1980,1981)$, RuINA (1980, 1983), Rice (1980), Kosloff and Liu (1980), Mavko(1980), and Rice and RUINA (1983). The latter authors comment that constitutive relations in the form of equations (1) provide a specific realization of a general class of frictional constitutive relations in which stress $\tau(t)$ at time $t$ is regarded as a direct function of instantaneous velocity $V(t)$ and as a functional of prior velocity $V\left(t^{\prime}\right),-\infty<t^{\prime}<t$.

The simplest and most commonly used model for discussion of slip motion is the spring-slider system shown in Fig. 1. It represents a single degree of freedom elastic dynamical system undergoing frictional slip at its contact with an adjoining body. A slider of mass $m$ (to be set equal to zero later for our quasi-static analysis) moves a distance $\delta$ at instantaneous rate $\dot{\delta}=V$. It is pulled by a load point at position $\delta_{0}$ moving at a rate $V_{0}$ by means of a spring with stiffness $k$. Within the context of this simple model some aspects of known stability results for the various friction laws are now summarized.

Analyses and observations show that slip motions may be roughly divided into two types: (1) those in which slip is nearly steady; and (2) those in which slip is intermittent. The simplest analysis is for the stability of steady sliding. In this case one examines the stability of motion with slip rate equal to load point displacement rate $\left(V=V_{0}\right)$, and the friction stress is (consequently) constant. For this analysis the only relevant classical friction law is one with friction dependent on slip rate, $\tau=\tau(V)$. A simple linear stability analysis reveals that steady sliding at $V_{0}$ is unstable if $\mathrm{d} \tau(V) / \mathrm{d} V<0$ at $V=V_{0}$ and is stable otherwise. A non-linear analysis reveals possible limit cycles near steady sliding if either $\mathrm{d} \tau(V) / \mathrm{d} V$ passes through zero for $V$ near $V_{0}$, or if an appropriately sized dashpot is placed parallel to the spring in Fig. 1 (BrockLEY and Ko, 1970; STOKER, 1950; HASSARD, KAZARINOFF and WAN, 1981). These limit cycles have a frequency of the same order as the natural vibration frequency of the spring and mass without friction.

Linear analysis of the stability of steady sliding with the state variable law of equation (1) reveals that steady sliding is stable if $\mathrm{d} \tau^{\mathrm{ss}} / \mathrm{d} V>0$, where $\tau^{\mathrm{ss}}(V)$ is the steady state value of $\tau$ for slip at constant $V$. For $\mathrm{d} \tau^{\mathrm{ss}} / \mathrm{d} V<0$ slip is stable for $k>k_{\text {er }}$ and unstable for $k<k_{\mathrm{cr}}$, where $k_{c s}$ is a finite critical stiffness (RICE and RUINA, 1983). At this critical stiffness the motion of the block is oscillatory. It is expected from general 
considerations of nonlinear stability (Hopf bifurcations, e.g. How ARD, 1979) that finite amplitude periodic motions will exist for some $k$ near $k_{\mathrm{cr}}$.

For friction governed by a single state variable $\theta[$ i.e. $\tau=F(V, \theta), \dot{\theta}=G(V, \theta)]$ the critical stiffness for steady slip at speed $V$ is (RICE and RUINA, 1983)

$$
k_{\mathrm{cr}}=-\frac{V}{L} \frac{\mathrm{d} \tau^{\mathrm{ss}}(V)}{\mathrm{d} V}\left[1+\frac{m V}{L \partial F(V, \theta) / \partial V}\right],
$$

evaluated at $\theta=\theta^{\mathrm{ss}}(V) ; L$ is the characteristic relaxation slip distance for the state variable $\theta$ [i.e. $L=-V /(\partial G(V, \theta) / \partial \theta)$ at $\theta=\theta^{\text {ss }}(V)$, so that the relation $\dot{\theta}=G(V, \theta)$ linearizes to $\mathrm{d} \theta / \mathrm{d} \delta=\left(\theta^{\text {ss }}-\theta\right) / L$ for $\theta$ near to $\left.\theta^{\text {ss }}(V)\right]$. The angular frequency of the oscillations at critical stiffness is given by

$$
\omega=\frac{V}{L}\left[\frac{-\mathrm{d} \tau^{\mathrm{ss}}(V) / \mathrm{d} V}{\partial F(V, \theta) / \partial V}\right]^{1 / 2}
$$

with $\theta=\theta^{\mathrm{ss}}(V)$, and the oscillations decay or grow in amplitude with time when $k$ is slightly greater or less, respectively, than $k_{\mathrm{cr}}$. For the rock experiments reported to date, the term in square brackets in (3) is of order unity, and for low slip rate $V$ it is clear that $\omega \approx V / L$ may be well less than $(k / m)^{1 / 2}$, the natural frequency of the spring-slider system. In this case it is straightforward to show that the terms within the square brackets in equation (2) differ negligibly from unity, i.e. the mass $m$ may be set to 0 , which is equivalent to the quasi-static analysis of motion in Ruins $(1980,1983)$. The linearized stability analysis will be discussed further subsequently.

On the other extreme from this nearly steady sliding is the intermittent slip termed 'stick-slip'. In stick-slip the block alternately sticks and slips. 'Slip' is modeled in elementary treatments to begin after 'stick' when the spring force reaches the 'static friction strength' which, depending on the model, may increase with the time of stationary contact or the rate at which the tangential load is applied. Once slip begins, the friction then (again, depending on the model) is either dependent on slip displacement or slip rate. When the integrated equations of motion predict that the slip rate falls back to zero, 'stick' resumes and the cycle repeats.

Since the state variable laws (1) are intended to describe a variety of slip histories, it should be possible to use them to model both the motion near steady state and the episodic motion with them. Dieterich $(1979 \mathrm{~b}, 1980,1981)$ and Mavko (1980) have done this numerically, neglecting inertia (although they simulate inertia indirectly for high slip rates) and have obtained results that resemble classical stick-slip.

The aim of this paper is to report some results, within the rate and state dependent constitutive framework, which begin to bridge the gap between a linearized description of stability near steady sliding and the description of fully developed 'stick-slip'. In particular, results are developed from general non-linear analysis of quasi-static motions of the spring-slider system, for a limited class of idealized friction laws suggested by experiment. The stability of the system is analysed when a steady slipping state is finitely perturbed, either by imposition of a sudden jump in load point displacement or velocity, or by holding the load point stationary for some 'relaxation' time before resuming imposed motion at the same speed. 


\section{Non-Linear State Variable Friction Laws}

\subsection{Constitutive relation with one state variable}

The simple one state variable constitutive law proposed by RuINA $(1980,1983)$ as representative of experiments on slip at constant normal stress $\sigma$ over a range of positive $V$, and motivated as an approximation to a more complex law by DIETERICH $(1979 \mathrm{a}, 1980,1981)$, is

$$
\begin{aligned}
\tau & =F(V, \theta) \equiv \tau_{*}+\theta+A \ln \left(V / V_{*}\right), \\
\mathrm{d} \theta / \mathrm{d} \iota & =G(V, \theta)=-(V / L)\left[\theta+B \ln \left(V / V_{*}\right)\right] .
\end{aligned}
$$

Here $V_{*}$ is an arbitrary positive constant and $\tau_{*}, A, B$ and $L$ are positive empirical constants with $\tau_{*}$ dependent on choice of $V_{*}$. Use of $L$ in (4) is consistent with its use in equations (2) and (3). In this expression, the frictional stress $\tau$ has a positive instantaneous rate dependence expressed by $A \ln \left(V / V_{*}\right)$, and the state variable $\theta$ evo ves towards the steady state value $-B \ln \left(V / V_{*}\right)$ with a characteristic slip distance $L$. The evolution with ongoing slip at a fixed $V$ is such that $\tau$ exponentially approaches the steady state value

$$
\tau^{\mathrm{ss}}=\tau^{\mathrm{ss}}(V) \equiv \tau_{*}-(B-A) \ln \left(V / V_{*}\right) .
$$

If $B>A$ as we assume here, this constitutes a long term negative rate dependence of $\tau: \mathrm{d} \tau^{\mathrm{ss}}(V) / \mathrm{d} V=-(B-A) / V<0$. One can observe from equation (4b) that the state variable $\theta$ varies continuously with time even when $\tau$ and $V$ vary discontinuously. This means that a sudden change in the stress $\tau$ causes a sudden change in slip velocity $V$ (or vice versa) and in $\mathrm{d} \theta / \mathrm{d} t$, but $\theta$ itself does not change discontinuously.

A response predicted by the above law is illustrated by Figs. $2 \mathrm{a}$ and $2 \mathrm{~b}$. Starting after steady state slip at speed $V_{0}$ and stress $\tau_{0}$, the frictional stress changes when the slip rate is suddenly increased by a factor $\mathrm{e} \cong 2.71828$ from $V_{0}$ to $\mathrm{e} V_{0}$. The frictional stress instantaneously increases to $\tau_{0}+A$, then falls exponentially with ongoing slip to a new steady state value $\tau_{0}-(B-A)$ over a characteristic slip distance $L$. The important features, verified at least approximately by experiments, are: (1) the frictional stress depends logarithmically on rate, both instantaneously and at steady state; and (2) at constant slip speed, the stress relaxes exponentially with a characteristic slip distance that is independent of slip rate.

We have fitted the one state variable law of equations (4) to DiETERICH's (1981) experiments on sliding of intact Westerly Granite blocks, with gouge layers of the same material between them, at $\sigma=100$ bars and speeds from 0.25 to $25 \mu \mathrm{m} / \mathrm{sec}$. The experiments varied gouge particle size, layer thickness and roughness of the intact rock surfaces. The surface roughness was the most significant variable, and separate fits for the roughest $(r)$ and smoothest ( $s$ ) of three surface preparations are: $A / \sigma=0.006$ to 0.008 for $(r)$ and 0.003 to 0.005 for $(s), B / A=1.12$ to 1.14 for $(r)$ and 1.5 to 2.3 for $(s)$, and $L=40$ to $50 \mu \mathrm{m}$ for $(r)$ and 4 to $25 \mu \mathrm{m}$ for (s). (A somewhat better fit of experimentally observed decay processes is given by a two state variable law described in the next subsection.)

Because later discussion for the one state variable law (4) will involve a 

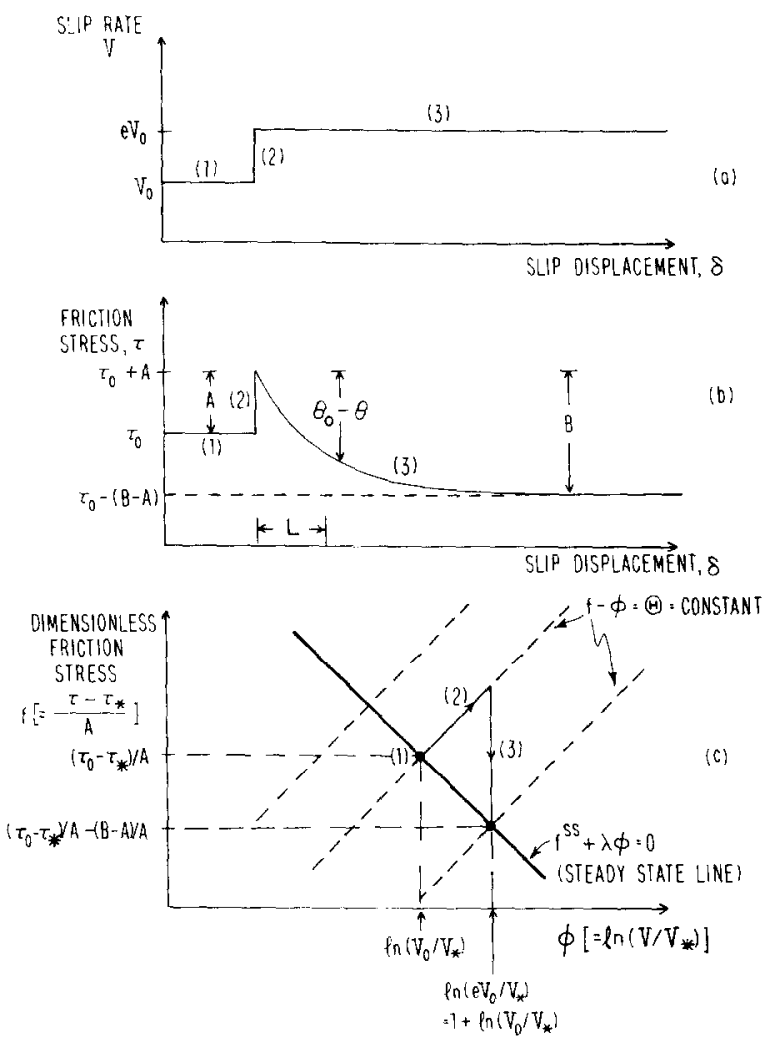

Fig. 2. Response to step change in slip rate at a sliding surface according to the friction law of equations (4). (a) The slip rate, constant at $V_{0}$ for a large slip distance (1), is suddenly changed to $V_{0}(2)$, and then held constant for a large slip distance (3). (b) The friction stress, constant at $\tau_{0}(1)$, suddenly increases by the amount $A$ at the jump in slip rate due to the instantaneous viscous-like response (2), and then decreases exponentially with ongoing slip (3) by amount $B$, with characteristic decay slip distance $L$, as the state variable evolves to its new steady state value. (c) The same response is plotted in a phase plane in terms of the variables $f\left[-\left(\tau-\tau_{*}\right) / A\right]$ and $\phi\left[=\ln \left(V / V_{*}\right)\right]$; the slip rate is initially constant at $V_{0}(1)$, then suddenly changes (2) so that $f$ and $\phi$ change along the dotted line of constant state, $\Theta=f-\phi=$ const., and then at the new constant slip rate (3) $f$ relaxes along the line $\phi=$ const. $(V=$ const. $)$ to the new steady state value.

non-dimensional phase plane, dimensionless quantities such as frictional stress $f \equiv\left(\tau-\tau_{*}\right) / A$, logarithmic velocity measure $\phi \equiv \ln \left(V / V_{*}\right)$, state variable $\Theta \equiv \theta / A$, constant $\beta \equiv B / A$ and time $T \equiv V_{*} t / L$ are introduced. Hence the non-dimensional form of the constitutive relation (4) can be rewritten as

$$
\begin{aligned}
f & =\Theta+\phi, \\
\mathrm{d} \Theta / \mathrm{d} T & =-\mathrm{e}^{\phi}(\Theta+\beta \phi)=-\mathrm{e}^{\phi}(f+\lambda \phi),
\end{aligned}
$$

where $\lambda \equiv(\beta-1) \equiv(B-A) / A$. At steady state, the block moves with the same speed as the load point (i.e. $\left.V=V_{0}\right), \phi=\ln \left(V_{0} / V_{*}\right)$ and $f=-\lambda \phi$. Therefore, a steady state motion with a particular load point speed can be represented by a point on a steady state line $f^{\text {ss }}+\lambda \phi=0$ in the $f, \phi$ phase plane as shown in Fig. $2 \mathrm{c}$. 
Recalling that the state $\Theta$ is always continuous, the experiment illustrated in Fig. $2 \mathrm{a}$ can be reproduced in Fig. $2 \mathrm{c}$ in terms of the variables $f$ and $\phi$. In steady state sliding with $V=V_{0}=$ const., $f=\left(\tau_{0}-\tau_{*}\right) / A$ and $\phi=\ln \left(V_{0} / V_{*}\right)$ are denoted by (1) in Fig. 2 . When the slip rate is suddenly increased to $V_{0}$ along $(2), \Theta=f-\phi$ remains constant. Thus the jumps in $f$ and $\phi$ are equal. The new slip rate $V$ is constant at $\mathrm{e} V_{0}$ (i.e. $\left.\phi=\ln \left(\mathrm{e} V_{0} / V_{*}\right)\right)$ and the frictional stress $f$ relaxes along (3) towards the new steady state value $f^{\mathrm{ss}}\left(\mathrm{e} V_{0}\right)=-\lambda \phi\left(\mathrm{e} V_{0}\right)=\left(\tau_{0}-\tau_{*}\right) / A-(B-A) / A$.

\subsection{Constitutive relation with two state variables}

The one state variable friction law (4) is a simple form representing the class of realistic state variable laws. It is used for discussion in this paper because it is mathematically simple. However, for experiments performed by DIETERICH (1981) and RUINA (1980, 1983), a two state variable law of similar structure provides a closer description of observed relaxations following jumps over a wide range (approximately, a factor of 100 ) of sliding speeds. This law has the form (R UINA, 1980, 1983), at constant normal stress $\sigma$,

$$
\begin{aligned}
\tau=F\left(V, \theta_{1}, \theta_{2}\right) & \equiv \tau_{*}+\theta_{1}+\theta_{2}+A \ln \left(V / V_{*}\right), \\
\mathrm{d} \theta_{1} / \mathrm{d} t=G_{1}\left(V, \theta_{1}, \theta_{2}\right) & \equiv-\left(V / L_{1}\right)\left[\theta_{1}+B_{1} \ln \left(V / V_{*}\right)\right], \\
\mathrm{d} \theta_{2} / \mathrm{d} t=G_{2}\left(V, \theta_{1}, \theta_{2}\right) & \equiv-\left(V / L_{2}\right)\left[\theta_{2}+B_{2} \ln \left(V / V_{*}\right)\right],
\end{aligned}
$$

where $V_{*}, \tau_{*}, A, B_{1}, B_{2}, L_{1}$ and $L_{2}$ are constants. Two examples of fits follow.

From experiments on polished quartzite surfaces sliding from 0.01 to $2 \mu \mathrm{m} / \mathrm{sec}$ at constant normal stress $\sigma=30$ bars, Ruina $(1980,1983)$ found $A / \sigma=0.011$, $B_{1} / A=1.00, B_{2} / A=0.84, L_{1} / L_{2}=0.048$, with $L_{1}=0.25 \mu \mathrm{m}$ and $L_{2}=5.2 \mu \mathrm{m}$. From experiments on Westerly Granite with $2 \mathrm{~mm}$ gouge layer, $<85 \mu \mathrm{m}$ particle size, between rough surfaces at constant normal stress $\sigma=100$ bars, we estimate (fitting figs 17 of DieTRICH, 1981) that $L_{1} / L_{2}=0.27$, with $L_{1}=20 \mu \mathrm{m}$ and $L_{2}=75 \mu \mathrm{m}$, $A / \sigma=0.015, B_{1} / A=0.67$ and $B_{2} / A=0.60$. Similar to the one state variable law, the frictional stress $\tau$ also has a positive instantaneous rate dependence denoted by $A \ln \left(V / V_{*}\right)$ and the state variables $O_{1}$ and $O_{2}$ evolve towards the steady state values $-B_{1} \ln \left(V / V_{*}\right)$ and $-B_{2} \ln \left(V / V_{*}\right)$ over characteristic distances $L_{1}$ and $L_{2}$ respectively. For sliding at constant speed, $\tau$ has a steady state value

$$
\tau^{\mathrm{ss}}(V)=\tau_{*}-\left(B_{1}+B_{2}-A\right) \ln \left(V / V_{*}\right)
$$

Again, if $B_{1}+B_{2}>A, \tau$ at steady sliding is a decreasing function of slip velocity $V$.

With the two state variable law (6), the illustration of the effect of a sudden jump in slip velocity as described earlier by Fig. $2 a, 2 b$ can be repeated and is shown in Fig. 3 . For the increase of slip rate by a factor of e, the frictional stress increases to $\tau_{0}+A$, as for the one state variable law in Fig. $2 \mathrm{~b}$, and the new steady state value is $\tau_{0}-\left(B_{1}+B_{2}-A\right)$. Figure $3 b$ shows the decay of the state variables with the corresponding characteristic slip distances. This two state variable law (6) is similar in form to equations (4), and reduces exactly to the one state variable law (4) when $L_{1}=L_{2}(=L)$ with $B=B_{1}+B_{2}$ and $\theta=\theta_{1}+\theta_{2}$. 


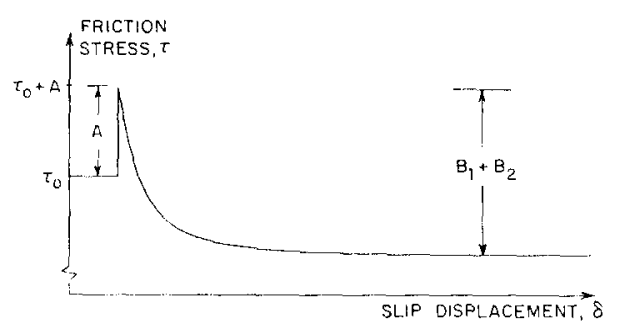

(a)

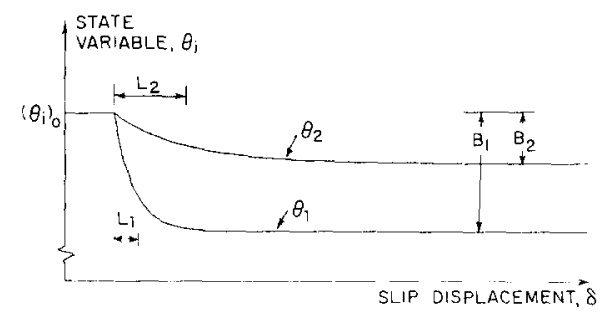

(b)
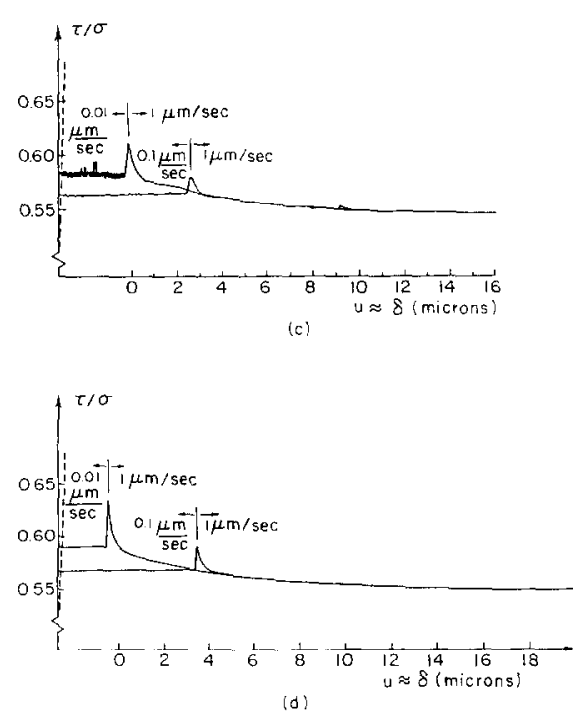

Fig. 3. Response to step change in slip rate for two state variable friction law of equations (6). (a) Stress response as the slip rate jumps from $V_{0}$ to $\mathrm{e} V_{0}$; the stress jumps to a peak $\tau_{0}+A$ from original steady state stress $\tau_{0}$, then decays exponentially with ongoing slip, with distinct decay slip distances $L_{1}$ and $L_{2}$, to the new steady state stress $\tau_{0}-\left(B_{1}+B_{2}-A\right)$. (b) Decay of the state variables $\theta_{1}, \theta_{2}$ to new steady state levels. (c) Experimental response to changes in slip rate on polished quartzite surfaces at normal stress $\sigma=30$ bars, from RUINA $(1980,1983) ; u$ is the relative displacement of a controlled set of measurement points close to the sliding surface, and $u \approx \delta$. (d) Simulation of experiments in (c) by two state variable friction law with $A=0.011 \sigma, B_{1} / A=1.00, B_{2} / A=0.84, L_{1}=0.25 \mu \mathrm{m}, L_{2}=5.2 \mu \mathrm{m}$.

Figure $3 c$ shows experimental results on polished quartzite by RuINA $(1980,1983)$ in two cases of suddenly imposed jumps in slip velocity $(u$ is the relative displacement of a controlled pair of points near to but not precisely on the slip surface; $u \approx \delta=$ the surface slip). Figure 3d shows Ruina's simulation of the experiments by the two state variable law of equations (6), using the parameters cited above.

In order to show clearly the short-slip decay process characterized by $L_{1}$ it is necessary that experiments accomplish a very abrupt, step-like increase in $V$. Otherwise, slip of order $L_{1}$ or greater may occur during the time taken to increase $V$ and the short-slip decay process is obscured. The two state variable law for the velocity jump test, in cases with $L_{1} \ll L_{2}$, would then appear as a one state variable law (with $A-B_{1}$ of the former identified as $A$ in the latter, and $B_{2}, L_{2}$ identified as $B, L$ ).

\section{Elastic Load System Interactions and Linear Stability Results}

The constitutive relation (4) or (6) describes the frictional stress at a sliding interface. In order to understand the behaviors of the one-degree-of-freedom system of Fig. 1, elastic load interaction must be considered simultaneously. We represent the elastic interactions by the linear spring with stiffness $k$ in series with the slider. The spring-force $\tau$ (per unit base area) is therefore related to motion of the slider and its load point by

$$
\tau=k\left(\delta_{0}-\delta\right), \quad \text { or } \mathrm{d} \tau / \mathrm{d} t=-k\left(V_{0}-V\right)
$$


where $\delta_{0}$ and $\delta$ are the load point and slider displacement respectively; inertia of the block is neglected here for simplicity. As mentioned earlier, the inclusion of inertia would not affect results in which the calculated acceleration is small.

For constant load point speed $V_{0}$, equations (6) (which include equations (4) as a special case) and (7), constitute a system of first order autonomous differential equations. A general analytic solution is not completely known. However, the simplest solution to this system of equations is the steady state solution:

$$
V=V_{0}, \quad \theta_{i}=\theta_{i}^{s s}=-B_{i} \ln \left(V_{0} / V_{*}\right), \quad \text { and } \quad \tau=\tau^{s s}=\tau_{*}-\left(B_{1}+B_{2}-A\right) \ln \left(V_{0} / V_{*}\right) \text {. }
$$

One may raise the question : will motion very near to this steady state motion be stable? From linear stability analysis, it is understood that if the real parts of the eigenvalues of the corresponding linear system of equations (6) and (7) are negative, zero, or positive, the solution will be stable, neutrally stable or unstable respectively.

In an equivalent approach, adequate for the entire class of constitutive relations represented by equations (1), RICE and RUINA (1983) regard the dependence of $\tau$ on surface state as being equivalent to the functional dependence of $\tau(t)$ on prior $V\left(t^{\prime}\right)$, $-\infty<t^{\prime}<t$, with loss of memory of slips in the distant past. They observe that the linearized expression for $\tau(t)$ in response to a perturbation $\dot{x}(t)$ of slip rate, such that $V(t)=V_{0}+\dot{x}(t)\left(|\dot{x}(t)| / V_{0} \ll 1\right)$, has the general form

$$
\tau(t)=\tau^{s s}+g \dot{x}(t)-\int_{0}^{t} h\left(t-t^{\prime}\right) \dot{x}\left(t^{\prime}\right) \mathrm{d} t^{\prime}
$$

assuming $\dot{x}(t)=0$ for $t<0$. Here $\tau^{\mathrm{ss}}=\tau^{\mathrm{ss}}\left(V_{0}\right), g$ and the memory function $h(t)$ depend on $V_{0}$, and

$$
\begin{gathered}
0<\left.g \equiv\left[\partial F\left(V, \theta_{1}, \theta_{2} \ldots\right) / \partial V\right]\right|_{V=V_{0}, \theta_{i}=\theta_{i}^{s s}\left(V_{0}\right)}, \\
\int_{0}^{\infty} h(t) \mathrm{d} t-g \equiv-\left.\left[\mathrm{d} \tau^{s s}(V) / \mathrm{d} V\right]\right|_{V=V_{0}} .
\end{gathered}
$$

As remarked following equation (1), experiments support the inequality in (9a). Data of DiETERICH $(1978,1979 \mathrm{a}, \mathrm{b}, 1981)$ and RUINA $(1980,1983)$ show that $\mathrm{d} \tau^{\mathrm{ss}}(V) / \mathrm{d} V<0$ in their experiments. However, experiments by Teurel (1981) indicate that $\mathrm{d} \tau^{\mathrm{se}}(V) / \mathrm{d} V$ ceases to remain negative at high $V$, as also is sometimes observed during early run-in periods and in experiments at high temperature.

RICE and RuINA (1983) prove that if $\mathrm{d} \tau^{\mathrm{ss}}\left(V_{0}\right) / \mathrm{d} V_{0}<0$, there exists a critical positive stiffness $k_{\mathrm{cr}}$ such that the steady slip state is stable for $k>k_{\mathrm{cr}}$ and unstable for $k<k_{\mathrm{cr}}$. In the vicinity of $k_{\mathrm{cr}}$, the system exhibits flutter oscillations of (circular) frequency $\omega$ whose amplitude grows in time if $k<k_{\mathrm{cr}}$ and decays if $k>k_{\mathrm{cr}}$. The critical frequency $\omega$ and critical stiffness $k_{\mathrm{cr}}$ are found by RICE and RUINA (1983) to be given by the real solution of

$$
\begin{gathered}
\int_{0}^{\infty} \cos (\omega t) h(t) \mathrm{d} t=g, \quad \text { and } \\
k_{\mathrm{cr}}=\omega \int_{0}^{\infty} \sin (\omega t) h(t) \mathrm{d} t .
\end{gathered}
$$


They show that if the decay in stress following step changes in slip rate is monotonic (i.e. $h(t)>0$ ), then $\mathrm{d} \tau^{\mathrm{ss}}\left(V_{0}\right) / \mathrm{d} V_{0}<0$ is a necessary and sufficient condition for the existence of a solution of equation (10a). Thus instability is possible, with some sufficiently reduced positive stiffness $k$, if friction stress decreases with slip rate at steady state.

If one linearizes the two state variables law as given by equations (6), and puts it in the functional form of equation (8), $g$ and $h(t)$ of (8) are found to be

$$
g=A / V_{0} \quad \text { and } \quad h(t)=\left(B_{1} / L_{1}\right) \mathrm{e}^{-V_{0} t / L_{1}}+\left(B_{2} / L_{2}\right) \mathrm{e}^{-V_{0} t / L_{2}}
$$

Equations $(10 \mathrm{a}, 10 \mathrm{~b})$ then give the critical frequency $\omega$ and critical stiffness $k_{\mathrm{er}}$ :

$$
\omega=\left[V_{0}(1+\rho) /\left(L_{1}+L_{2}\right) \sqrt{\rho}\right] \sqrt{\left[2 \kappa_{\mathrm{cr}}-\left(\beta_{1}+\beta_{2}-1\right)\right]}
$$

and

$$
\begin{aligned}
\kappa_{\mathrm{cr}} \equiv k_{\mathrm{cr}}\left(L_{1}+L_{2}\right) / 2 A= & {\left[\left(\beta_{1}-1\right)+\rho^{2}\left(\beta_{2}-1\right)+2 \rho\left(\beta_{1}+\beta_{2}-1\right)\right.} \\
& \left.+\sqrt{ }\left\{\left[\left(\beta_{1}-1\right)+\rho^{2}\left(\beta_{2}-1\right)\right]^{2}+4 \rho^{2}\left(\beta_{1}+\beta_{2}-1\right)\right\}\right] / 4 \rho
\end{aligned}
$$

where $\rho=L_{1} / L_{2}$ and $\beta_{1,2}=B_{1,2} / A$. If $\rho=1\left(L_{1}=L_{2}=L\right)$ and $\beta=\beta_{1}+\beta_{2}$, the corresponding critical frequency $\omega$ and critical stiffness $\kappa_{\mathrm{cr}}$ for the one state variable law are obtained,

$$
\begin{gathered}
\omega=\left(V_{0} / L\right) \sqrt{ }(\beta-1) \equiv\left(V_{0} / L\right) \sqrt{\lambda}, \\
\kappa_{\mathrm{cr}}=k_{\mathrm{cr}} L / A=\beta-1 \equiv \lambda .
\end{gathered}
$$

Equations (13a) and (13b) can also be equivalently deduced from equations (3) and (2), with $m=0$. The constant $\lambda$, which appears often in what follows, can be equated to the bracketed term in (3). Some representative values of $\beta_{1}, \beta_{2}, \rho$ and the associated $\kappa_{\mathrm{cr}}$ calculated from (12b) are listed in Table 1.

The linearized stability analysis shows that small perturbations of slip in steady state

TABLE 1. Examples of the critical spring stiffness $k_{\mathrm{cr}}$ in steady state slip, according to linear stability theory, for the two state variable friction law of equations $(6) ; \kappa_{\mathrm{cr}}$ calculated from equation (12b)

\begin{tabular}{cccc}
\hline$\beta_{1} \equiv B_{1} / A$ & $\beta_{2} \equiv B_{2} / A$ & $\rho \equiv L_{1} / L_{2}$ & $\kappa_{\mathrm{cr}} \equiv k_{\mathrm{cr}}\left(L_{1}+L_{2}\right) / 2 A$ \\
\hline any value & any value & 1 & $\beta_{2}+\beta_{2}-1$ \\
1 & 1 & any value & 1 \\
1.00 & 0.84 & 0.048 & $0.876 \dagger$ \\
0.67 & 0.60 & 0.27 & $0.224 \ddagger$ \\
1.3 & 0.5 & 0.20 & 1.318 \\
1.5 & 1.2 & 0.20 & 2.395 \\
0.2 & 1.5 & 0.20 & 0.436 \\
1.0 & 1.5 & 0.20 & 1.388
\end{tabular}

† From Ruina $(1980,1983)$ data on polished quartzite surfaces.

† From DiETERICH (1981) data on Westerly granite with $2 \mathrm{~mm}$ gouge layer, $<85 \mu \mathrm{m}$ particle size, between rough intact rock surfaces. 
cause sustained periodic sinusoidal oscillations when $k=k_{\mathrm{cr}}$, but cause oscillations with amplitude that either decays or grows exponentially with time when $k$ is slightly greater or less, respectively, than $k_{\mathrm{cr}}$. Such response defines a Hopf bifurcation (e.g. HoWARD, 1979; HASSARD, KAZARINOFF and WAN, 1981) at $k=k_{\mathrm{cr}}$. The theory of the Hopf bifurcation then shows that finite amplitude periodic solutions exist for values of $k$ in some sufficiently small neighborhood of $k_{\mathrm{cr}}$. It may be that this occurs for $k>k_{\mathrm{cr}}$ (in which case the periodic solutions are unstable), for $k<k_{\text {cr }}$ (stable limit cycles) or, exceptionally (but as occurs in our one state variable case), for $k=k_{c r}$.

A nonlinear stability analysis using the Hopf technique was done by hand for the one state variable law and numerically (using the computer program by HASSARD, KAZARINOFF and WAN, 1981) for the two state variable law. In short the procedure may be described by introducing a vector

$$
\{z\}=\left\{\tau \quad \tau^{\mathrm{ss}}\left(V_{0}\right), \theta_{1}-\theta_{1}^{s s}\left(V_{0}\right), \theta_{2}-\theta_{2}^{s s}\left(V_{0}\right), \ldots\right\},
$$

having the properties that $\{z\}=\{0\}$ in the steady slip state examined and that, according to a linearized description of motion of the spring-slider system, periodic solutions

$$
\{z\}=\text { const. } \times \operatorname{Re}\left[\left\{z_{L}\right\} \mathrm{e}^{i \omega t}\right]
$$

exist at $k=k_{\mathrm{cr}}$, where $\omega$ and $k_{\mathrm{cr}}$ are given by equations (10), and $\left\{z_{L}\right\}$ is a mode shape vector. One then seeks exact oscillatory solutions to the non-linear equations for the spring-slider system, of small finite amplitude (parameterized by $\varepsilon$ ), in the form

$$
\{z\}=\varepsilon \operatorname{Re}\left[\left\{z_{L}\right\} \exp \left(i \omega\left(1+\xi_{2} \varepsilon^{2}+\cdots\right) t\right)\right]+\mathrm{O}\left(\varepsilon^{2}\right),
$$

where $O\left(\varepsilon^{2}\right)$ represents time dependent periodic terms of the order indicated or higher in $\varepsilon$ and $\xi_{2}$ is an unknown term showing the first non-zero perturbation of the oscillation period. Such periodic solutions are found generally to exist for amplitudedependent stiffnesses given by an expansion of the form

$$
k=k_{\mathrm{cr}}\left(1-\mu_{2} \varepsilon^{2}-\cdots\right) \text {. }
$$

Here the sign of $\mu_{2}$, whose calculation is the object of the Hopf technique, determines whether the solutions exist for either $k>k_{\text {cr }}\left(\mu_{2}<0\right.$ and periodic solutions are unstable) or $k<k_{\text {cr }}\left(\mu_{2}>0\right.$ and limit cycle solutions are stable). Hence the analysis gives the curvature of the $\varepsilon$ vs $k$ locus of periodic solutions at $\varepsilon=0$.

The results are indicated in plots of $\varepsilon$ vs $\kappa$ (dimensionless $k$ ) by the curvature of the solid line segments in Fig. $4 \mathrm{a}$ for the one state variable law and Fig. $4 \mathrm{~b}$ for the two state variable law. Remarkably, the curvature is zero $\left(\mu_{2}=0\right)$ for the one state variable case and, as indicated by the straight dashed line, a full analysis (next section) shows that finite amplitude oscillations are found at $\kappa=\kappa_{\mathrm{cr}}$ for a range of the amplitude parameter $\varepsilon$. The dashed line is shown bifurcated abruptly to the right at a certain point. This occurs because (as will be clear from the next figure) motions with $\kappa=\kappa_{\mathrm{cr}}$ that begin sufficiently far from steady state are unstable, in that $V \rightarrow \infty$ in finite time. This transition from periodic to unstable motions at $\kappa=\kappa_{\mathrm{cr}}$ may, in fact, occur at an unbounded amplitude as measured by $\varepsilon$, but it occurs at a finite amplitude as measured by physical variables (e.g. by $\tau_{\max }-\tau^{s s}\left(V_{0}\right)$, where $\tau_{\max }$ is the maximum stress in the motion). This is the reason for the appended question mark in Fig. 4a, which indicates 


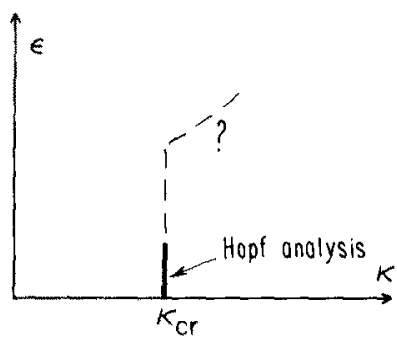

(o)

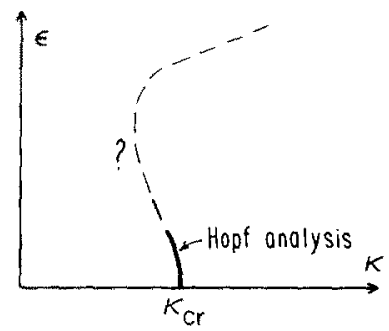

(b)

ONE STATE VARIABLE

FIG. 4. Solid line segments illustrate results of Hopf bifurcation analysis for (a) one state variable and (b) two state variable friction law. The segments show how the dimensionless spring stiffness $\kappa$ must vary from $\kappa_{\mathrm{cr}}$ (its critical value according to linear stability theory) in order for finite amplitude periodic solutions, with amplitude measured by $\varepsilon$, to exist in response to motion of the load point at uniform speed. The one state variable result in (a) is exceptional and persists over a range of finite amplitudes as shown by the dashed line continuation of the solid segment. Curvature exhibited for the two state variable case in (b) shows the existence of stable limit cycle oscillatory solution in a small range of stiffnesses $\kappa$ below $\kappa_{\mathrm{cr}}$ (see Figs. 13 and 14 for further discussion). Dashed line segments with appended question marks are intended only as qualitative indications of response at larger amplitudes; see text.

that the segment of dashed line extending to the right is only a qualitative indicator of response type. This extension of the dashed line to the right indicates that for $\kappa>\kappa_{\mathrm{cr}}$, there exists a boundary at sufficiently large amplitude between oscillatory motions with $V-V_{0} \rightarrow 0$ and motions with $V-V_{0} \rightarrow \infty$. This boundary is a locus of unstable periodic solutions.

For the two state variable friction law, the curvature of the $\varepsilon$ vs $\kappa$ relation at $\varepsilon=0$, Fig. $4 b$, is always of a sign (except for special parameter values reducing it to the one state variable law) so as to assure the existence of stable limit cycle oscillations in some sufficiently small neighborhood $\kappa<\kappa_{\mathrm{cr}}$. Our numerical integrations of equations (6) and (7) suggest a response qualitatively like the dashed line continuation of the $\varepsilon$ vs $\kappa$ locus (the question mark again denotes its imprecision; among other possibilities it may bifurcate into a family of loci). The dashed line is intended to indicate that periodic solutions are found only in a small range of $\kappa$ below $\kappa_{\mathrm{cr}}$ and that response is comparable to that of the one state variable case when $\kappa>\kappa_{c r}$. More is reported on this at the end of the paper.

\section{Non-Linear Analysis of SPring-Sllder System witil One State Variable Friction Law}

Here we adopt the dimensionless variables $f, \phi$ and $\Theta$ introduced to write the one state variable friction law as in equations (5), and used also in Fig. 2c. In terms of these, the last version of equation ( 7 ) is

$$
\mathrm{d} f / \mathrm{d} T=\kappa\left(v_{0}-\mathrm{e}^{\phi}\right)
$$

where $\kappa=k L / A$ and $v_{0}=V_{0} / V_{*}$. Combining equations (5) with this, we obtain

$$
\mathrm{d} \phi / \mathrm{d} T=\mathrm{e}^{\phi}(-\kappa+f+\lambda \phi)+\kappa v_{0} .
$$


Equations (14) represent the system of non-linear equations governing quasistatic motions of a single degree of freedom elastic system following the one state variable friction law. We note that solutions to equations (14) obey a scaling rule. That is, if $f_{1}(T)$ and $\phi_{1}(T)$ are solutions corresponding to load-point speed $v_{0}$ then $f=f_{1}(s T)-\lambda \ln s$ and $\phi=\phi_{1}(s T)+\ln s$ is a solution corresponding to load point speed $s v_{0}$. The scaling rule can also be justified by noting that $v_{0}=V_{0} / V_{*}$ and $V_{*}$ may be chosen arbitrarily. The rule applies more generally in using solutions corresponding to load-point motion $\delta_{u}(t)$ to generate those which correspond to $\delta_{0}(s t)$, and a similar rule applies to motions governed by the two state variable friction law (RUINA, 1980). Consequently, if $v_{0}>0$ is a constant imposed load-point speed, we may take $v_{0}=1$ and not lose any features of solutions or stability criteria. Related to this is the lack of dependence of critical stiffness $\kappa_{\mathrm{er}}$ on $v_{0}$ in equations (12b) and (13b). We do not replace $v_{0}$ with 1 in all subsequent calculations since we will look at solutions with step changes in $v_{0}$.

The trajectories in the $f-\phi$ phase plane (like that in Fig. 2 c) for the system considered are given in differential form by eliminating time $T$ from equations (14) to obtain

$$
0=\left[(-\kappa+f+\lambda \phi) \mathrm{e}^{\phi}+\kappa v_{0}\right] \mathrm{d} f-\kappa\left(v_{0}-\mathrm{e}^{\phi}\right) \mathrm{d} \phi .
$$

The right side of (15) is not a perfect differential but it expresses the fact that $\mathrm{d} P(f, \phi)=0$ where $P=$ const. is the solution being sought. Multiplying (15) by an integrating factor which can be written in the form $\mathrm{e}^{q(f, \phi)}$, we have

$$
\mathrm{d} P=\mathrm{e}^{q(f, \phi)}\left[(-\kappa+f+\lambda \phi) \mathrm{e}^{\phi}+\kappa v_{0}\right] \mathrm{d} f-\mathrm{e}^{q(f, \phi)} \kappa\left(v_{0}-\mathrm{e}^{\phi}\right) \mathrm{d} \phi=0 .
$$

Denoting the coefficients of $\mathrm{d} f$ and $\mathrm{d} \phi$ in (16) as $P_{f}$ and $P_{\phi}$ respectively, the integrating factor must allow satisfaction of $\partial P_{f} / \partial \phi=\partial P_{\phi} / \partial f$, which then requires that

$$
[(1+\partial q / \partial \phi)(-\kappa+f+\lambda \phi)+\lambda-\kappa \partial q / \partial f] \mathrm{e}^{\phi}+\left[(\partial q / \partial \phi+\partial q / \partial f) \kappa v_{0}\right]=0 .
$$

This equation is generally not easy to solve for $q$ but once it is solved, the function $P$ can be found and $P=$ const. represents a path or orbit in the $f, \phi$ phase plane which the system follows.

\subsection{An integral for $\kappa=\lambda \equiv \kappa_{\mathrm{cr}}$ (stiffness is equal to that for linear neutral stability)}

In order to understand the general motion predicted for this one state variable law, a special case, $\kappa=\kappa_{\mathrm{cr}} \equiv \lambda$, is considered first. By inspection, $q=f-\phi$ is a solution to equation (17) in this case. One then integrates (16) to obtain trajectories in the form

$$
P_{1} \equiv \mathrm{e}^{f}\left[f+\kappa\left(\phi+v_{0} \mathrm{e}^{-\phi}-1\right)-1\right]=\mathrm{const} .
$$

At a steady state $\phi=\ln v_{0}$ and $f^{\mathrm{ss}}=-\lambda \ln v_{0}$, so that $P_{1}^{\mathrm{ss}}=-v_{0}^{-\lambda}$. Curves of $P_{1}=$ constant are shown as solid lines in Fig. 5 for $\lambda=1$ (a representative value from the fits to experiments mentioned earlier) and $v_{0}=1$, involving no loss of generality as mentioned. Therefore, at steady state, $P_{1}^{s s}=-1$. If $-1<P_{1}<0$ (i.e. the system deviates slightly from steady state) the orbits are closed curves, which shows that the system exhibits finite amplitude periodic oscillations. Since all these orbits occur at the fixed value $\kappa=\lambda$, the vertical dashed line in Fig. 4 a is justified. $P_{1}>0$ corresponds to open trajectories extending to infinity and represents unstable motions. Therefore, $\Gamma_{1}=0$ gives the limiting trajectory separating (periodic) stable and unstable orbits. 


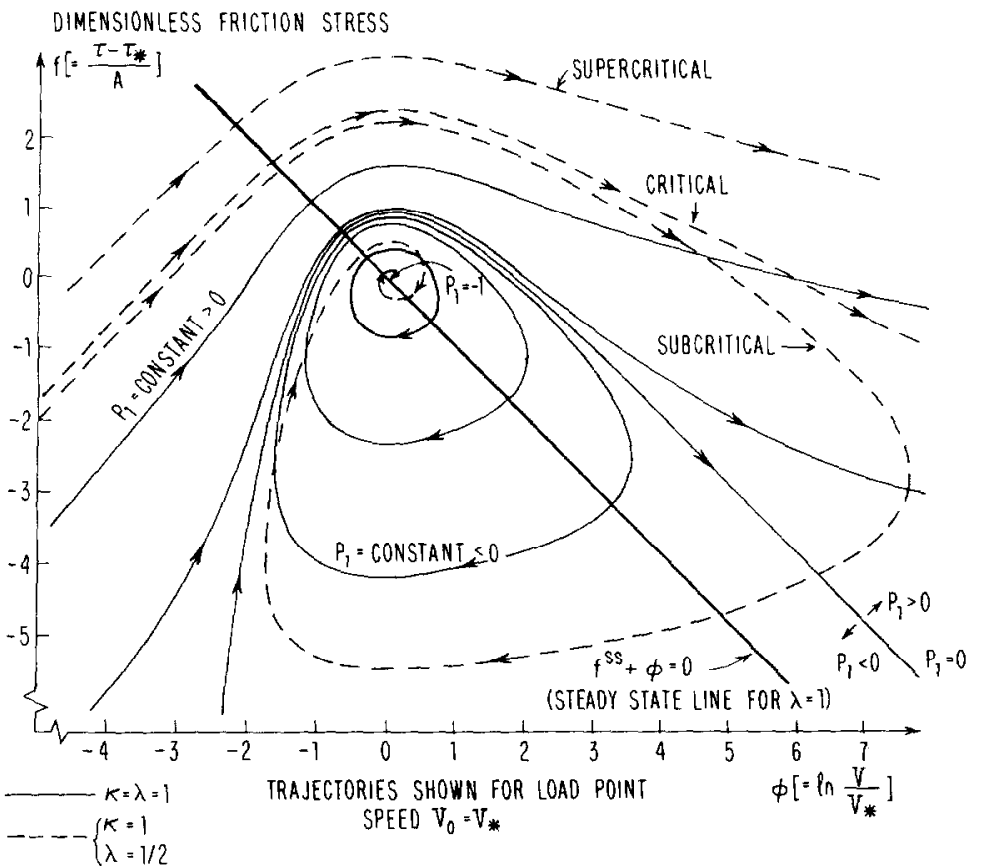

FIG. 5. Phase plane of dimensionless friction stress $f\left[=\left(\tau-\tau_{*}\right) / A\right]$ and velocity measure $\phi\left[=\ln \left(V / V_{*}\right)\right]$ for constant rate load point motion at $V_{0}$, chosen as $V_{0}=V_{*}$. Solid lines are trajectories for stiffness $\kappa=\kappa_{c r} \equiv \lambda$, with $\lambda=1$. These trajectories are the level lines of $P_{1}$ in equation (18); $P_{1}<0$ are closed orbits, $P_{1}>0$ are unstable orbits on which $V \rightarrow \infty$. Heavy solid line is steady state relations between $f^{\text {ss }}$ and $\ln \left(V / V_{*}\right)$. Dashed lines are orbits for $\kappa=1, \kappa_{\mathrm{cr}} \equiv \lambda=\frac{1}{2}$. The subcritical dashed orbit decays to steady sliding, the supercritical orbit leads to infinite slip rates, and a critical orbit separates the two types. The solid curves provide level lines of a Liapunov function for general motions with $\kappa \neq \lambda$.

\subsection{General analysis of motion by Liapunov method}

The function $P_{1}$ is also useful as a Liapunov function for cases in which the spring stiffness $\kappa$ is different from the critical value, $\lambda$. Indeed, if one identifies a related function $P^{\prime}$ by

$$
P^{\prime}=\mathrm{e}^{f}\left[f+\kappa\left(\phi+v_{0} \mathrm{e}^{-\phi}-1\right)-1+(\lambda-\kappa) \ln v_{0}\right]
$$

and uses the identity

$$
\mathrm{d} P^{\prime} / \mathrm{d} T=\left(\partial P^{\prime} / \partial f\right)(\mathrm{d} f / \mathrm{d} T)+\left(\partial P^{\prime} / \partial \phi\right)(\mathrm{d} \phi / \mathrm{d} T)
$$

and equations (14), one finds that

$$
\mathrm{d} P^{\prime} / \mathrm{d} T=\mathrm{e}^{f} \kappa(\lambda-\kappa)\left(\phi-\ln v_{0}\right)\left(\mathrm{e}^{\phi}-v_{0}\right) .
$$

Since $\left(\phi-\ln v_{0}\right)\left(\mathrm{e}^{\phi}-v_{0}\right)$ is always positive for $\phi \neq \ln v_{0}$, we have

$$
\mathrm{d} \boldsymbol{P}^{\prime} / \mathrm{d} T<0 \text { for } \kappa>\lambda
$$

and

$$
\mathrm{d} P^{\prime} / \mathrm{d} T>0 \text { for } \quad \kappa<\lambda
$$


We now explain why $P^{\prime}$ can be regarded as a Liapunov function by first deducing the geometry of curves $P^{\prime}(f, \phi)=$ constant in the $f, \phi$ phase plane. We note that the global minimum of the function $P^{\prime}(f, \phi)$ occurs at the steady state point $\phi=\ln v_{0}, f=-\lambda \ln v_{0}$, and $P_{\min }^{\prime}=-v_{0}^{-\lambda}$. Now we show that curves $P^{\prime}=$ const., with $P_{\text {min }}^{\prime}<P^{\prime}<0$, are closed orbits in the $f, \phi$ plane. This is shown by observing that, for given $f, P^{\prime}=$ const. (in the above range) gives exactly two values of $\phi$, and that for a given $\phi, P^{\prime}=$ const. gives exactly two values of $f$. Rearranging equation (19) gives

$$
\widetilde{P}(\phi) \equiv \kappa\left(v_{0} \mathrm{e}^{-\phi}+\phi\right)=P^{\prime} \mathrm{e}^{-f}-f+1+\kappa-(\lambda-\kappa) \ln v_{0} .
$$

If we fix $P^{\prime}$ and $f$, then $\mathrm{d}^{2} \tilde{P}(\phi) / \mathrm{d}^{2} \phi=\kappa v_{0} \mathrm{e}^{-\phi}>0$, so that if the system is not at steady state, there are two values for $\phi$ satisfying (22). Likewise, if one rearranges equation (22) such that

$$
\hat{P}(f) \equiv P^{\prime} \mathrm{e}^{-f}-f=\kappa\left(v_{0} \mathrm{e}^{-\phi}+\phi\right)-1-\kappa+(\lambda-\kappa) \ln v_{0}
$$

and fixes $P^{\prime}$ and $\phi$, then $\mathrm{d}^{2} \hat{P}(f) / \mathrm{d} f^{2}=P^{\prime} \mathrm{e}^{-f}<0$ and, again, if the system is not at steady state, there are two values for $f$ satisfying (23). Hence $P^{\prime}=$ const., with $P_{\min }^{\prime}<P^{\prime}$ $<0$, is a closed path in the $f, \phi$ phase diagram, and the path degenerates to a point, which is the steady state point, when $P^{\prime}=P_{\text {min }}^{\prime}$.

Next, we show that if one moves away from the steady state point, $P^{\prime}$ increases monotonically. This follows by calculating from (19) that

$$
\partial P^{\prime} / \partial \phi=\left(\mathrm{e}^{\phi}-v_{0}\right) \kappa \mathrm{e}^{f-\phi},
$$

so that $\partial P^{\prime} / \partial \phi$ has the same sign as $\phi-\ln v_{0}$. We therefore conclude that $P^{\prime}$ is a suitable Liapunov function, in that it defines a family of closed curves with $P^{\prime}$ increasing monotonically as one crosses curves of the family moving away from the steady state point at which $P^{\prime}=P_{\min }^{\prime}$. Pictorially, the properties described are summarized by the solid lines $P_{1}=$ constant in Fig. 5 and, indeed, the function $P^{\prime}(f, \phi)$ is identical to $P_{1}(f, \phi)$ when we choose $v_{0}=1$ as in that figure.

The inequalities (21) thus insure that, for $\kappa>\lambda$, orbits are constantly crossing curves $P^{\prime}=$ const. in a direction so as to decrease $P^{\prime}$. Hence, when $\kappa>\hat{\lambda} \equiv \kappa_{\mathrm{cr}}$, any orbit which passes within the region of the phase plane covered by closed curves of type $P^{\prime}=$ constant will be drawn towards the steady state point $\phi=\ln v_{0}, f=-\lambda \ln v_{0}$ at which $P^{\prime}=P_{\min }^{\prime}$. This shows that the steadily slipping state is stable to sufficiently limited finite perturbations when $\kappa>\lambda$. The numerically computed dashed orbits in Fig. 5 correspond to $\kappa=1, \lambda=0.5, v_{0}=1$, and verify the result. The (subcritical) orbit which passes into the domain of closed curves is drawn to their center at the steady state point. Another (supercritical) orbit remains far from the closed curves and corresponds to unstable behavior with $\phi \rightarrow \infty$; a critical dividing orbit between the stable and unstable regimes is also shown. By contrast, equation (21b) shows that when $\kappa<\lambda \equiv \kappa_{\mathrm{cr}}$ the steady state point is always unstable since orbits will then follow paths which increase $P^{\prime}$.

For $\phi$ large and negative $(\phi \rightarrow-\infty), k$ and $\lambda$ arhitrary and $v_{0} \neq 0$, equation (14) immediately shows that $f=\kappa v_{0} T+$ const., and $\phi=\kappa v_{0} T+$ const. are solutions. They appear as the $45^{\circ}$ solutions on the left edge of Fig. 5. These are constant state trajectories. That is, for very small $v$ (large negative $\phi=\ln v$ ) the state changes very 


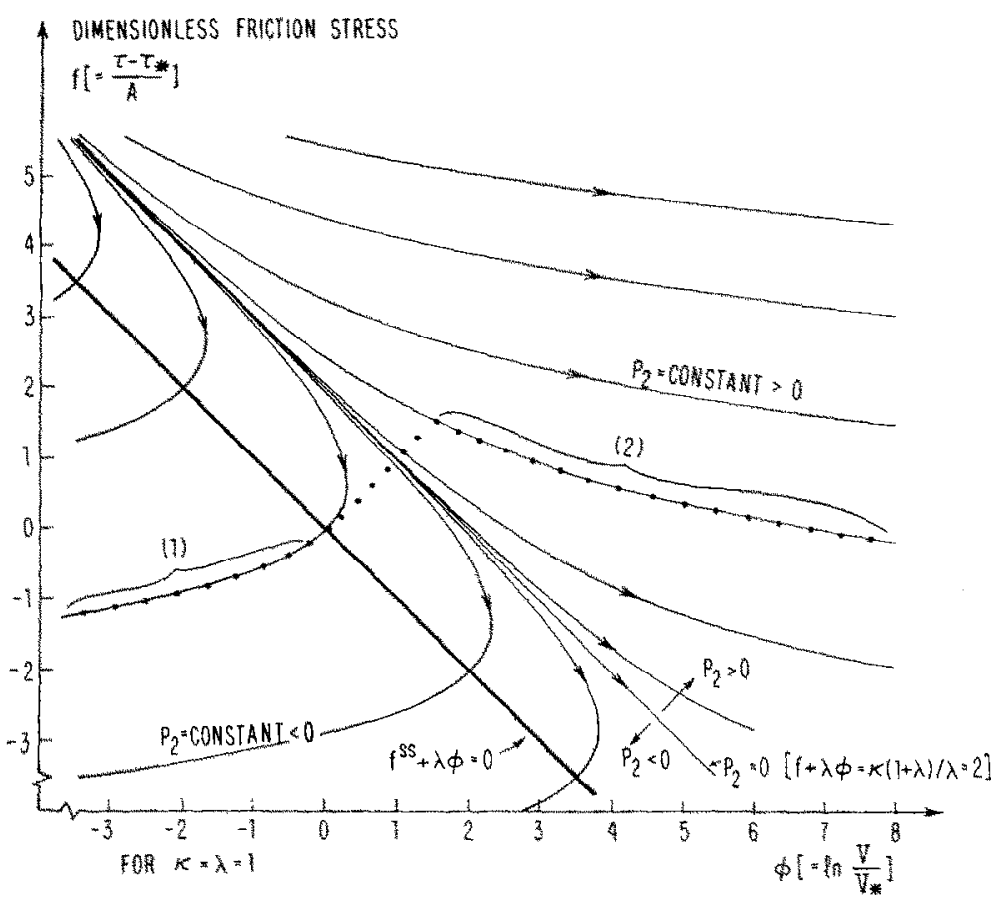

Fig. 6. Trajectories of slider motion for a stationary load point, $V_{0}=0$, shown for the case $\lambda=\kappa=1$. The curves are level lines of the function $P_{2}$ in equations (25) and the curve $P_{2}=0$ separates unstable orbits $\left(P_{2}>0\right)$ from stable orbits $\left(P_{2}<0\right)$. The dotted curve $(1)$ shows the trajectory followed if steady load point motion at $V_{0}=V_{*}$ is suddenly stopped. The dotted curve (2) shows the motion if the load point, after steady motion at rate $V_{0}=V_{*}$, suddenly makes a dispiacement jump and then stops. A supercritical jump is shown.

slowly. The friction stress change is due only to spring stretch from load point motion. The slip rate change is due only to the viscous part of the friction law ( $A$ in equation (4)).

As remarked, for $\kappa>\kappa_{\mathrm{cr}}$ the system is stable, at least for small but finite perturbations. One may then ask for what kind of perturbations and what magnitude will the system be stable? Further insight is provided by study of the following special case.

\subsection{Analysis of stationary load point}

The exact solution (18) for trajectories is limited to $k=\lambda$. We were able to solve other than numerically for trajectories with arbitrary $\lambda$ and $k$ only when the load point is stationary, $v_{0}=0$. In that case equation (17) is solved by $q=\lambda f / \kappa-\phi$ and the solution trajectories are given by

$$
P_{2} \equiv \mathrm{e}^{(\lambda / /) / f}[(f+\lambda \phi)-\kappa(1+\lambda) / \lambda]=\text { const. }
$$

They are shown in Fig. 6 as solid lines, drawn for the case $x=\lambda=1$. Solving (25) for $\phi$ we obtain

$$
\phi=\left[P_{2} \mathrm{e}^{-(\lambda / \kappa) / f}-f+\kappa(1+\lambda) / \lambda\right] / \lambda .
$$


The time derivative of $P_{2}$ in equation (25) is, using equation (14) for general non-zero $v_{0}$,

$$
\mathrm{d} P_{2} / \mathrm{d} T=\mathrm{e}^{(\lambda / \kappa) f} v_{0} \lambda(f+\lambda \phi),
$$

which is of course zero for $v_{0}=0$. Also, applying (26) to (14) with $v_{0}=0$ one can solve for

$$
\mathrm{d} f / \mathrm{d} T=-\kappa \exp \left\{\left[P_{2} \mathrm{e}^{-(\lambda / \kappa) f}-f+\kappa(1+\lambda) / \lambda\right] / \lambda\right\}<0 .
$$

Thus, although we cannot integrate (28) explicitly for the time history of $f$, we can see from (28) (or directly from (14)) that $f$ decreases along trajectories (as shown by dotted lines (1) and (2) in Fig. 6). This merely states that the spring relaxes if the load point is still.

For large negative $\phi$ the stable orbits $\left(P_{2}<0\right)$ are described by equation (26) and the lower branch asymptotically by

$$
\mathrm{d} f / \mathrm{d} T=-\kappa \exp \left[\left(P_{2} / \lambda\right) \mathrm{e}^{-(\lambda / \kappa) f}\right] .
$$

Equation (29) implies that, eventually, $f$ decreases much more slowly than logarithmically with time during stationary holds.

The straight line $P_{2}=0$, corresponding to $f+\lambda \phi=\kappa(1+\lambda) / \lambda(=2$ in Fig. 6$)$, is the dividing trajectory (so long as $\lambda>0$ ) between solutions for which $\phi(\equiv \ln v)$ decays $\left(P_{2}<0\right)$, and extremely unstable $(\phi \rightarrow \infty)$ solutions $\left(P_{2}>0\right)$.

The solution (25) has some implications even when $v_{0} \neq 0$. Since for $P_{2}=0$, the dividing trajectory, $f+\lambda \phi>0$, equation (27) states that $\mathrm{d} P_{2} / \mathrm{d} T>0$ for $v_{0}>0$. Thus the full solution to equations (14), with arbitrary $\kappa, \lambda>0$ and $v_{0}$ necessarily leads to instabilities if the trajectory crosses above $f+\lambda \phi=\kappa(1+\lambda) / \lambda$.

The solution (25) to equations (14) with $v_{0}=0$ can also be viewed as the asymptotic solution for a case of large positive $\phi$, i.e. large speeds $v \gg v_{0}$, such that $v_{0}$ can be neglected in equation (17). For $P_{2}>0$ (i.c. $f+\lambda \phi>\kappa(1+\lambda) / \lambda$ ), equation (26) then gives the asymptotic solution

$$
\phi=P_{2} \mathrm{e}^{-(\lambda / \kappa) f} / \lambda, \quad f=-(\kappa / \lambda) \ln \left(\lambda \phi / P_{2}\right)
$$

which is, approximately, on the right edge of Figs. 5 and 6. Applying equation (29) to equations (14) we have the asymptotic result for unstable orbits $\left(P_{2}>0\right)$ that as $\phi \rightarrow \infty$

$$
\mathrm{d} \phi / \mathrm{d} T=\lambda \phi \mathrm{e}^{\phi} .
$$

Since $\int_{\phi>0}^{\infty}\left(\mathrm{e}^{-\phi^{\prime}} / \phi^{\prime}\right) \mathrm{d} \phi^{\prime}$ is bounded, $\phi \rightarrow \infty$ in finite time. In short, for all $\lambda, \kappa, v_{0}>0$, any solution crossing above the line $f+\lambda \phi=\kappa(1+\lambda) / \lambda$ leads to infinite slip speed in finite time (as noted in RuINA $(1980,1983)$ for the limit $\kappa=0$ ).

\section{Perturbations of a Steadily Sliding System, One State Variable Friction LaW}

In this section, three types of perturbations to steady state sliding will be discussed: (1) the load point is suddenly displaced and then held stationary; (2) the load point velocity is suddenly increased; (3) the load point is held stationary for some 'relaxation' 


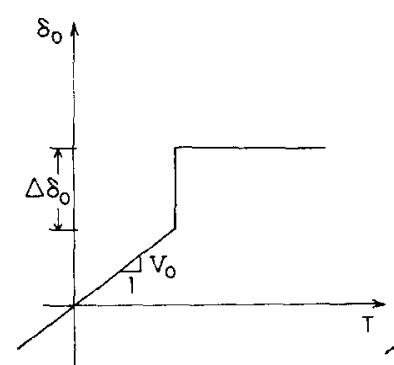

(a) SUDDEN LOAD-POINT DISPLACEMENT JUMP

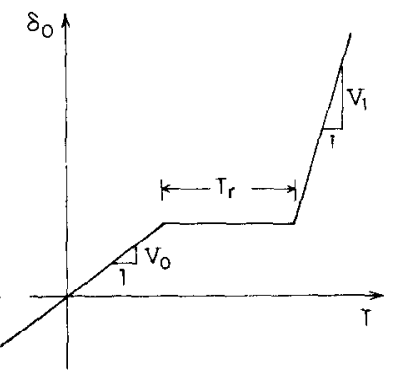

(b) RELAXATION FOR Tr AND RESUMPTION OF DIFFERENT

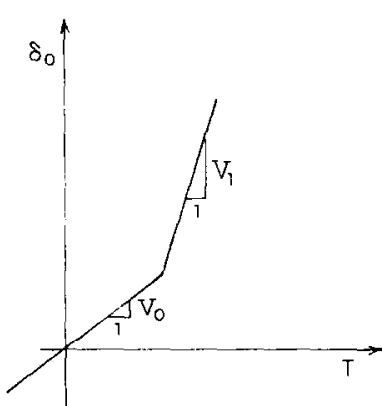
LOAD SPEED

(c) SUDDEN LOAD POINT VELOCITY JUMP

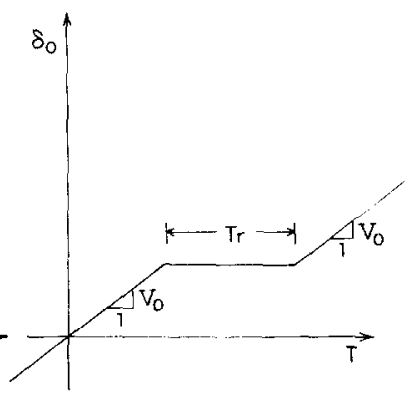

(d) RELAXATION FOR Tr AND RESUMPTION OF THE SAME LOAD POINT SPEED

FIG. 7. Schematic diagram showing four kinds of perturbations: (a) sudden jump in loadpoint displacement, which is then held fixed; (b) stopping of load point motion for relaxation time $T_{\mathrm{r}}$ and resuming motion at different load point speed $V_{1} ;$ (c) sudden jump in load point velocity; (d) stopping of load point for relaxation time $T_{\mathrm{r}}$ and resuming the same speed.

time and its motion is then resumed at the original speed. The stability results will be obtained by patching together solutions from the preceding sections using appropriate jump conditions and also by numerical integration.

In the first case, Fig. $7 \mathrm{a}, \Delta(f-\phi)=0$ during the jump, since there is no jump in state $\theta$ when the load point jumps. Therefore, in order to jump $f+\lambda \phi=\kappa(1+\lambda) / \lambda$ (to cross the instability trajectory for $v_{0}=0$ ) from the steady state line $f+\lambda \phi=0$, along a line of constant state $\Theta=f-\phi$, requires that $\Delta f=\Delta \phi=\kappa / \lambda$. This means the instantaneous jump in the slip rate is to $v_{1} / v_{0}-V_{1} / V_{0}=-\mathrm{e}^{\kappa / \lambda}$ (where here $V_{0}$ and $V_{1}$ are the slip rate just before and after the jump), and $\Delta \delta_{0}=L / \lambda$ is the jump in load point displacement necessary to cause the instability. When $\kappa=\lambda$, for example, the minimum jump in load point displacement required to cause massive instability, $\Delta \delta_{0}=L / \lambda$, also causes an instantaneous slip rate change by a factor of $e \cong 2.71828$. An unstable jump across the dividing line in Fig. 6 is shown, leading to unstable slip on the dotted trajectory labelled (2) that begins on the steady state line before the jump.

We discuss the second and third cases together with reference to Fig. 7b. Suppose the system begins in steady state sliding with load point speed $v_{0}=V_{0} / V_{*}$ (which gives $\left.v=v_{0}, \phi=\ln v_{0}, f^{\mathrm{ss}}=-\lambda \phi\right)$. The load point is then stopped $\left(v_{0}=0\right)$ for some relaxation time $T_{\mathrm{r}}$. At this stage, the stress begins to relax and the velocity of the slider drops rapidly according to the spring force law (7) and the state $\theta$ increases slowly (because of 
the low speed). After the relaxation time $T_{\mathrm{r}}$, load point motion is resumed at a new speed $v_{1}\left(=V_{1} / V_{*}\right.$, where $V_{1}$ is the new load point velocity).

We consider the case $\kappa=\lambda \equiv \kappa_{\mathrm{cr}}$. Let the arbitrary constant $V_{*}$ be $V_{0}$ (initial point velocity) so that the steady state solution is $v_{0}=1, f=\phi=0$, and $P_{1}=-1$. In the relaxation stage, $v_{0}=0$ and the solution can be represented by $P_{2}$ in equation (25), with $\kappa$ set equal to $\lambda$. The value of $P_{2}$ is found by substituting the initial values of $f$ and $\phi$ $(f=\phi=0)$. Thus

$$
P_{2} \equiv \mathrm{e}^{f}[f+\lambda \phi-(1+\lambda)]=-(1+\lambda)
$$

so that $f$ and $\phi$ are related by

$$
f+\lambda \phi=(1+\lambda)\left(1-\mathrm{e}^{-f}\right) .
$$

In the reloading stage, the load point moves with the new speed $v_{1}$ and trajectories are given by equation (18) with $v_{1}$ written for $v_{0}$. Denoting $\Theta_{\mathrm{r}}$ as the value to which the state parameter has risen at the end of the relaxation stage $(\Theta=0$ at the start of relaxation), and remembering that $\Theta=f-\phi$ and using (33), we find initial conditions for the reloading stage, during which

$$
P_{1} \equiv \mathrm{e}^{f}\left[f+\lambda\left(\phi+v_{1} \mathrm{e}^{-\phi}-1\right)-1\right]=-(1+\lambda)+\lambda v_{1} \mathrm{e}^{\boldsymbol{\theta}_{\mathrm{x}}} \text {. }
$$

As was pointed out earlier, $P_{1}=0$ is the boundary between stable and unstable solutions when $\kappa=\lambda$. The critical combinations of relaxation effects and reloading speeds for instability in this case are therefore given by

$$
\left(V_{1} / V_{0}\right) \mathrm{e}^{\Theta_{\mathrm{r}}}=(1+\lambda) / \lambda .
$$

If the relaxation time $T_{\mathrm{r}}=0$ (then $\Theta_{\mathrm{r}}=0$ ) the perturbation corresponds to a sudden jump in load point speed as shown in Fig. 7c. The response is therefore unstable if $V_{1} / V_{0}$ $>(1+\lambda) / \lambda$ for the case $\kappa=\lambda$. For $\kappa>\lambda$, the critical velocity jump below which the solution is stable is found numerically by integrating equations (14). Some examples (for the case $\lambda=1$ ) of stress vs slip response and related trajectories in the $f, \phi$ phase diagram are shown in Figs. 8 and 9 respectively. For $\kappa=\lambda$, the system exhibits finite amplitude periodic oscillations for a velocity jump $V_{1} / V_{0}$ less than $(1+\lambda) / \lambda(=2$ in this case), Fig. 8a. For $\kappa>\lambda$ and $V_{1} / V_{0}$ near to but less than the critical velocity jump (subcritical), the friction stress oscillates initially but quickly evolves to the new steady state level, Fig. 8b. For $\kappa>\lambda$ and $V_{1} / V_{0}$ greater than the critical velocity jump (supercritical), the friction stress drops rapidly towards $-\infty$ (unstable), Fig. 8c. For $\kappa>\lambda$ and $V_{1} / V_{0}$ far less than the critical value (strongly subcritical), the friction stress drops rapidly to the new steady state value immediately after the instantaneous increase, Fig. 8 d. This is similar to Fig. 2 b. Figure 9 shows the $f, \phi$ phase plane with a closed orbit for the case with $\kappa=\lambda$, an inward spiral for $\kappa>\lambda$ with subcritical velocity jump, an open trajectory for supercritical velocity jump, and a fairly direct path for the strongly subcritical case (similar to the path 2, 3 in Fig. 2c).

Numerically computed stability boundaries for the type of perturbation in Fig. $7 \mathrm{c}$ are shown in Fig. 10, where the critical load point velocity jump $\left(V_{1} / V_{\mathrm{o}}\right)_{\mathrm{cr}}$ is plotted against normalized spring stiffness $\kappa / \lambda\left(=\kappa / \kappa_{\mathrm{cr}}=k / k_{\mathrm{cr}}\right)$ for various values of $\lambda$. Each solid line divides finite amplitude perturbation regions (above and to the left), giving unstable response ( $V \rightarrow \infty$ in finite time), from regions giving a stable response (below and to the 


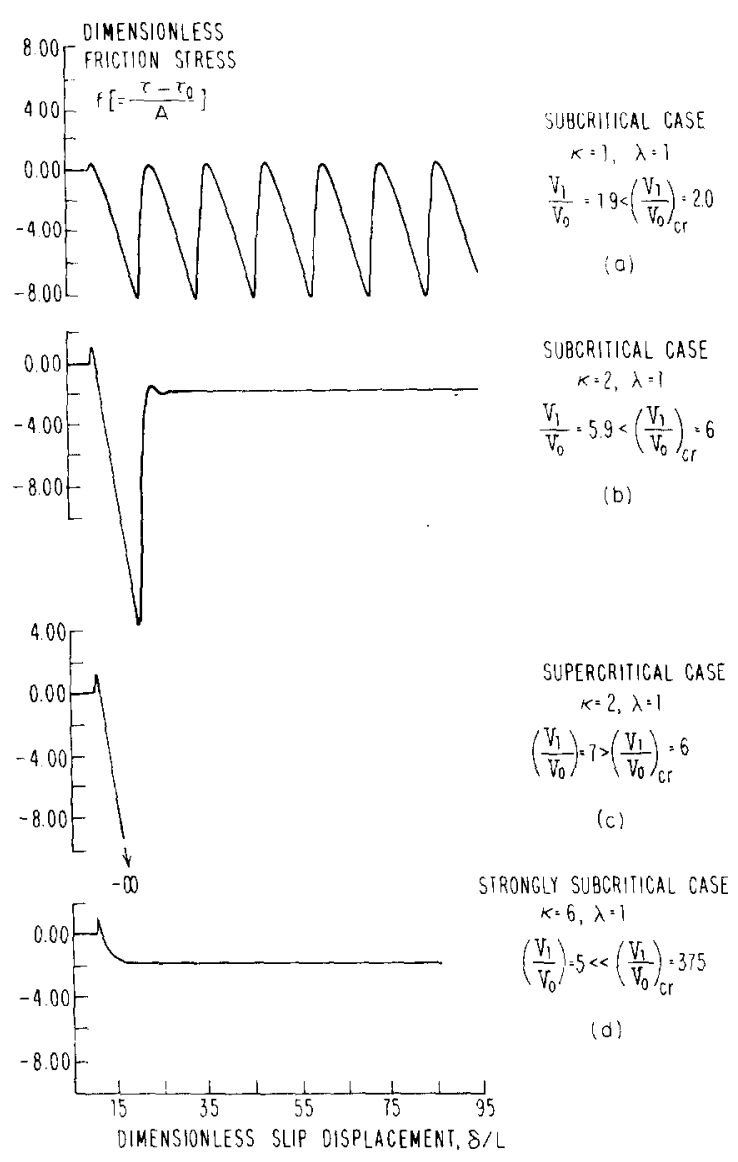

F1G. 8. Stress response due to load point velocity jump ratio $V_{1 /} / V_{0}\left(V_{1}=\right.$ new load point velocity, $V_{0}$ $=$ original load point velocity); $\kappa_{\mathrm{cr}} \equiv \lambda=1$ in all cases. (a) Suhcritical case $k=1, V_{1} / V_{0}=1.9<\left(V_{1} / V_{0}\right)_{\mathrm{cr}}$ $=2$. (b) Subcritical case $k=2, V_{1} / V_{0}=5.9<\left(V_{1} / V_{0}\right)_{c r}=6$. (c) Supercritical case $k=2, V_{1} / V_{0}=7$ $>\left(V_{1} / V_{0}\right)_{\mathrm{er}}=6$. (d) Strongly subcritical case $\kappa=6, V_{1} / V_{0}=5 \ll\left(V_{1} / V_{0}\right)_{\mathrm{er}}=375$.

right) in which the system evolves to a new steady state consistent with the new load point speed $V_{1}$. The vertical segment of each solid line in Fig. 10 , rising from $\kappa / \lambda=1$, is the locus of perturbations giving sustained periodic response. These vertical segments correspond to the vertical dashed line in Fig. $4 \mathrm{a}$, and the sudden deviation of that dashed line to the right is meant to reflect the corresponding deviations of the stability boundaries in Fig. 10. In the next section we examine a stability diagram like Fig. 10 for the two state variable case and find that it has a similar but somewhat richer structure. The dashed lines in Fig. 10 are apparent asymptotes, $(1 / \lambda) \mathrm{e}^{\kappa / \lambda}$, of the solid lines. We note as a curiosity that when $\lambda=e-1$, the apparent asymptote and numerically found solid line coincide.

DIETERICH (1978), in arguing for the importance of spring stiffness in stick-slip, has presented a qualitative analysis applicable to the case just studied. His reasoning, applied to the friction law considered here, is as follows. In changing the slip rate from 


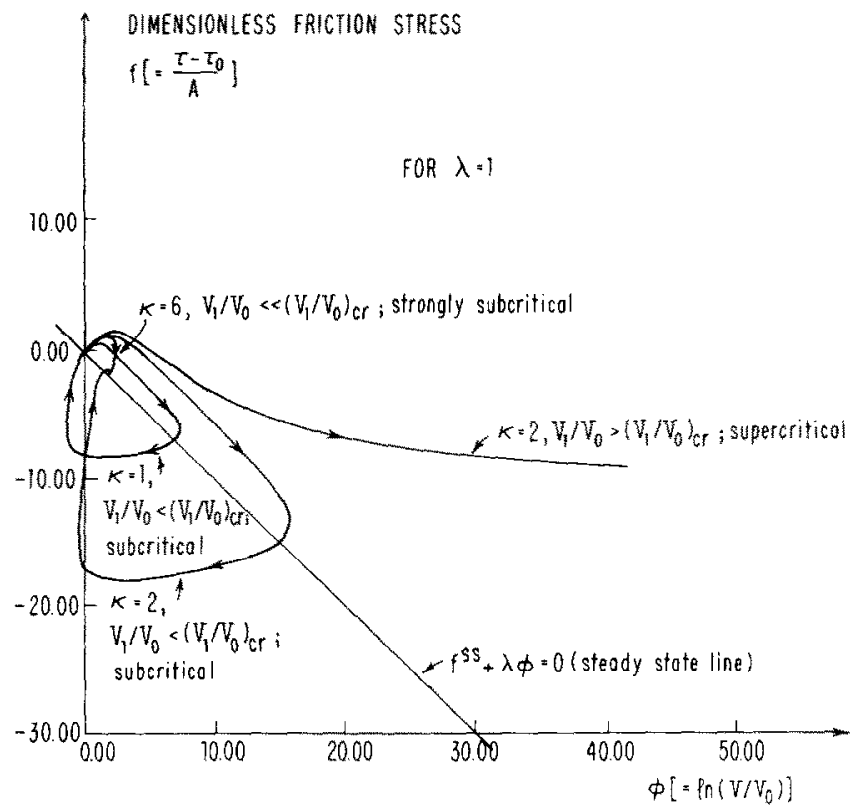

FiG. 9. The $f, \phi$ phase diagram for the cases described in Fig. 8. Closed orbit (permanent oscillation) for $\kappa=\lambda$ and subcritical velocity jump. Inward spiral to new steady state for subcritical velocity jump and $k>\lambda$. Open trajectory for supercritical velocity jump. Direct path to new steady state solution for strongly subcritical velocity jump.

$V_{0}$ to $V_{1}$ the friction force decreases by $(B-A) \ln \left(V_{1} / V_{0}\right)$. This drop occurs roughly over the slip distance $L$. If the friction force drops more rapidly with slip than the spring force, slip is predicted to be unstable. Dieterich's predicted stability boundary is thus $(B-A) \ln \left(V_{1} / V_{0}\right)_{\mathrm{cr}} / L=k$ or $\left(V_{1} / V_{0}\right)_{\mathrm{cr}}=\mathrm{e}^{\kappa / \lambda}$. This result is close to ours for $\lambda \cong 1$ and $\kappa>\lambda_{\text {. }}$ (His analysis was performed without recognition of the direct rate dependence and thus corresponds roughly to $A \rightarrow 0$ and $\lambda \rightarrow \infty$ ). For $\kappa<\lambda$ this reasoning leads to the incorrect result that finite perturbations are required for instability.

When $T_{\mathrm{r}}>0$ and $V_{1} / V_{0}=1$, the situation corresponds to Fig. $7 \mathrm{~d}$. In this case, and with $\kappa=\lambda$, the system will become unstable if $\mathrm{e}^{\Theta_{\mathrm{r}}}>(1+\lambda) / \lambda$. Since the state $\Theta_{\mathrm{r}}$ at the end of the relaxation stage is a monotonic increasing function of $T_{\mathrm{r}}$, the instability of the system can be regarded alternatively as governed by $T_{r}$. That is, if $T_{\mathrm{r}}$ is greater than a critical value $T_{\mathrm{cr}}$, which is in turn related to the quantity $\ln (1+1 / \lambda)$, the system becomes unstable; otherwise, it is stable. The critical relaxation times $T_{\mathrm{cr}}$ with different $\kappa$, not necessarily equal to $\lambda$, have been found numerically and are plotted in Fig. 11. For $T_{\mathrm{r}}$ in the regions below and to the right of the solid lines, the system is stable and evolves again towards steady state when motion of the load point resumes. For $T_{\mathrm{r}}$ values in the regions above and to the left of the solid lines, the system is unstable with $V \rightarrow \infty$ in finite time. The asymptotes of the solid lines are not fully known but seem to be in the form $T_{\mathrm{r}}=n \mathrm{e}^{m \kappa}$ where $m=m(\lambda)$ and $n=n(\lambda)$.

Figure 12 shows some numerically computed responses of stress vs sliding distance for different relaxation times and stiffnesses. As described by DIETERICH (1981), during 


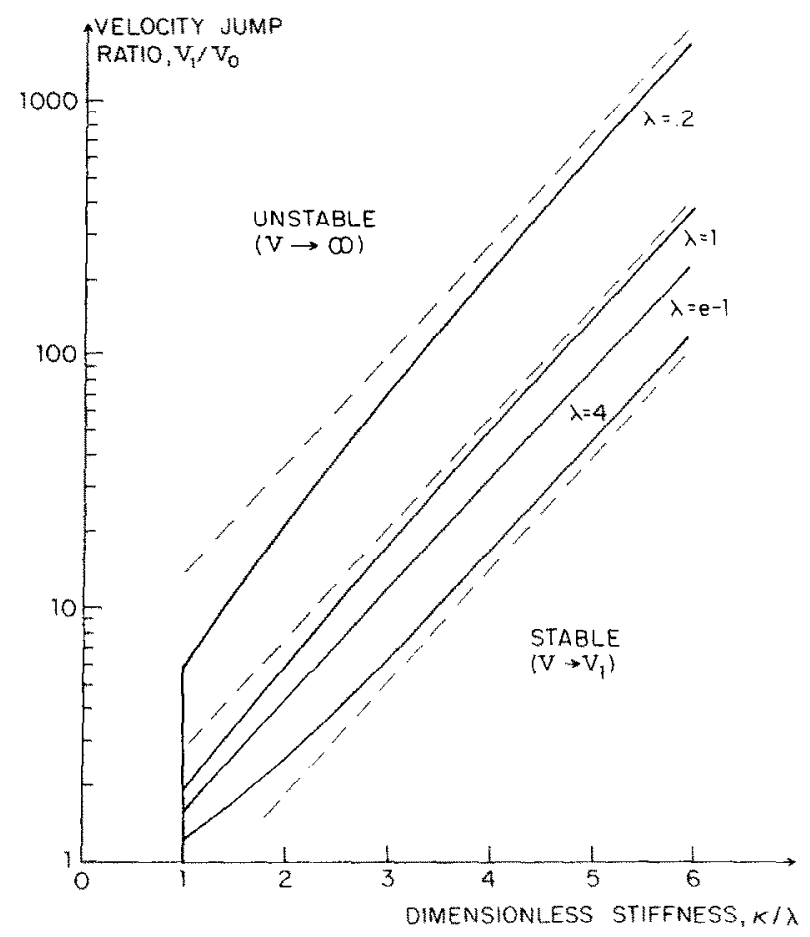

Fig. 10. Stability boundaries for load point velocity jump perturbations as in Fig. 7c. Each solid line shows the critical value of $V_{1} / V_{0}$ as a function of spring stiffness $\kappa$, for the value of $\lambda \equiv \kappa_{\mathrm{cr}}$ indicated on that line. At $\kappa / \lambda=1,\left(V_{1} / V_{0}\right)_{\mathrm{cr}}=(1+\lambda) / \lambda$ as deduced in the text. The dashed lines show apparent asymptotes given by $V_{1} / V_{0}=(1 / \lambda) \mathrm{e}^{\kappa / \lambda}$. For $\lambda=\mathrm{e}-1 \simeq 1.71828$, the apparent asymptote coincides with the solid curve. Perturbations above and to left of the boundary line cause $V \rightarrow \infty$ in finite time. Those below and to the right cause an approach to the new steady state at speed $V_{1}$.

relaxation, the friction stress relaxes according to the spring force law, giving a slope $-\kappa$ in the stress vs slip plot. At the end of relaxation, the load point resumes the original speed. This sudden step increase in load point speed causes a rapid increase of stress and slip rate. Afterwards, as the slip progresses, the negative dependence of state on speed causes the stress $f$ to decay, leading either to instability or to a possibly oscillatory return to the original steady state stress.

The reason for the appearance of a strength peak can be seen from equations (4). During relaxation, $V<V_{0}$ and hence $\theta$ evolves continuously (and incompletely) towards values $\theta^{\text {ss }}(V)>\theta^{\text {ss }}\left(V_{0}\right)$. Since $V<V_{0}$ not only during relaxation but also during the resumed motion of the load point, so long as $i>0$ (as is evident from equations (7)), we have that $\theta>\theta^{\text {ss }}\left(V_{0}\right)$. Hence to again reach steady state it is necessary that $\dot{\tau}$ pass through zero (i.e. that $\tau$ exhibit a strength peak) and since $V=V_{0}$ at the peak by equations ( 7$)$, we have the result

$$
\tau_{\text {peak }}-\tau^{\mathrm{ss}}\left(V_{0}\right)=\theta_{\text {peak }}-\theta^{\mathrm{ss}}\left(V_{0}\right)>0 .
$$




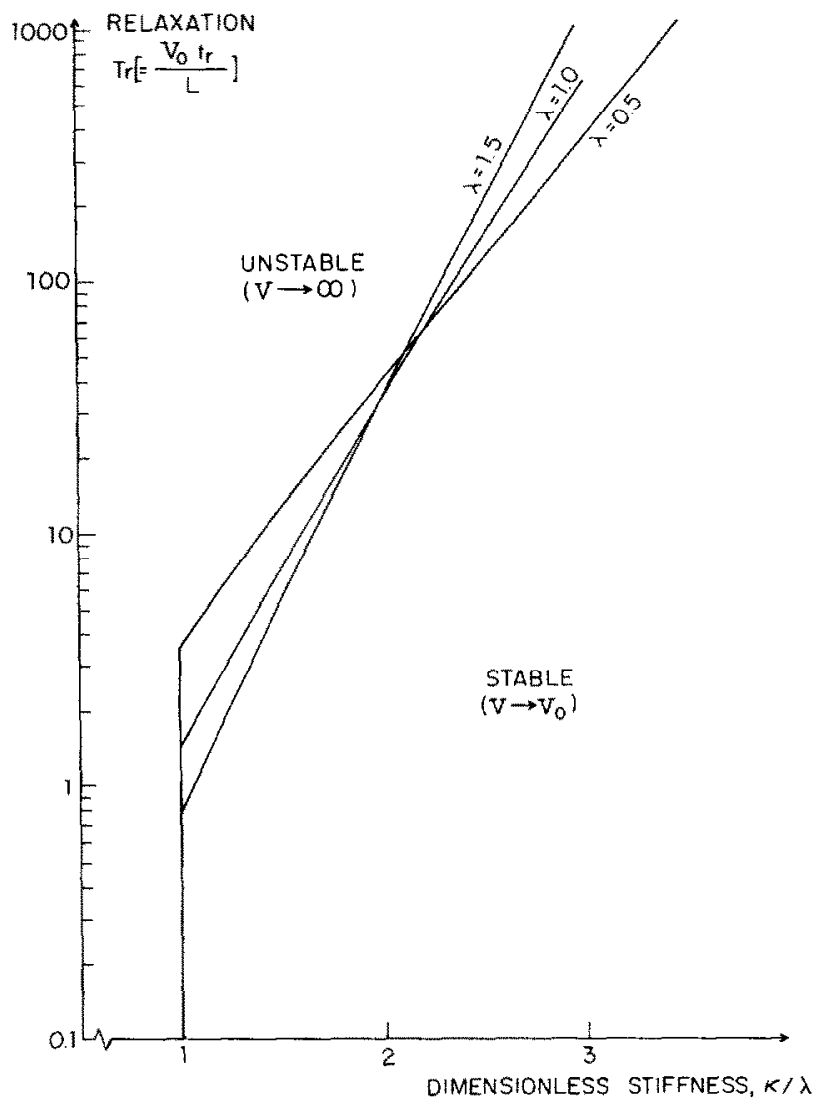

FIG. 11. Critical dimensionless relaxation time $T_{\mathrm{r}}$ vs $\kappa / \lambda$ for perturbation as in Fig. $7 \mathrm{~d}$. Regions above and to left of the curves are unstable $(V \rightarrow \infty$ in finite time) while regions below and to the right the curves are stable (return to steady state). The apparent asymptotes are of the form $T_{\tau}=n(\lambda) \mathrm{e}^{m(\lambda) \kappa}$.

\section{Perturbations, Two State Variable Friction Law}

Comparison of Fig. $4 \mathrm{~b}$ with $4 \mathrm{a}$ suggests that the responses to perturbations will be somewhat more complicated for the two than for the one state variable friction law. It is not possible to present as comprehensive a picture of results for the two variable case as done here for the one state variable law. We have, however, done extensive numerical integrations of equations (6) and (7) for a spring-slider system with a two state variable friction law, beginning in steady state and perturbed as in Fig. $7 \mathrm{c}$ by sudden increase in load point velocity from $V_{0}$ to $V_{1}$.

Figure 13 shows a stability boundary plot, analogous to that in Fig. 10, summarizing results of the numerical integrations (for the parameters $\beta_{1} \equiv B_{1} / A=1.00, \beta_{2} \equiv B_{2} / A$ $=0.84$, and $\rho \equiv L_{1} / L_{2}=0.048$ ). In the region marked 'unstable' the slip speed becomes unbounded in finite time, and in that marked 'stable' the perturbed system evolves towards the new steady state at speed $V_{1}$. However, between these regions there 


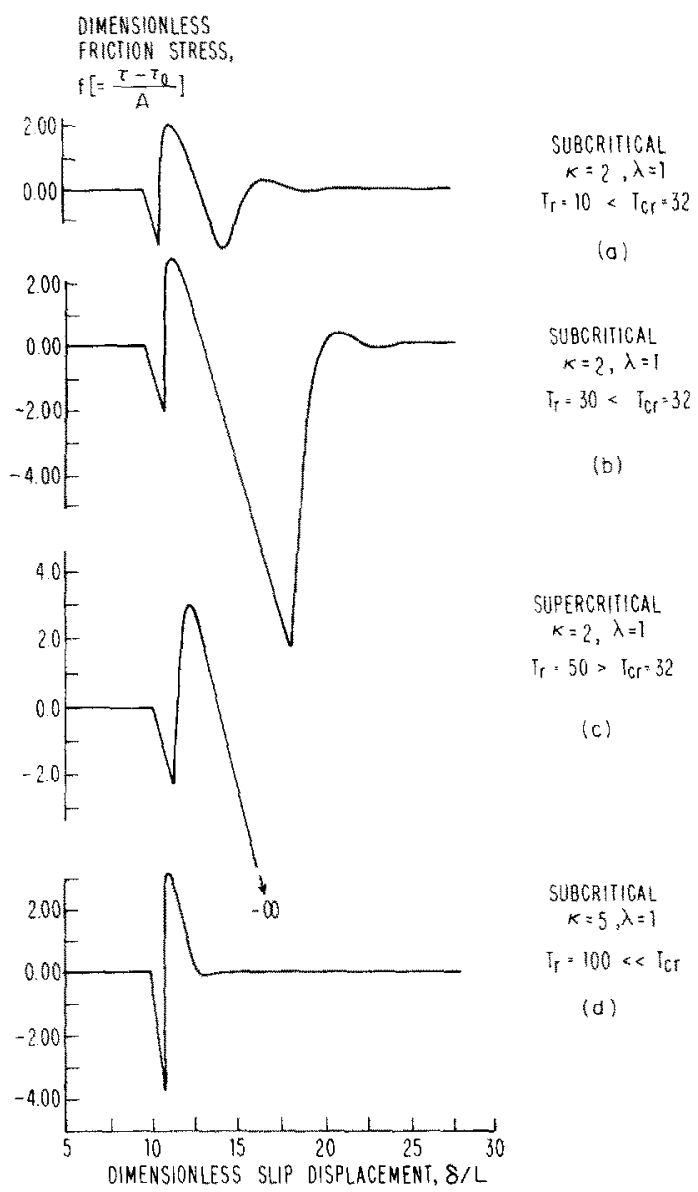

Fig. 12. Friction stress response due to relaxation and resumption of load point motion at initial speed. The slope of the curve during the relaxation is $-\kappa$ (spring constant). (a) Subcritical, (b) marginally subcritical, and (c) supercritical for $\kappa=2$ and $\lambda=\kappa_{\mathrm{cr}}=1$. (d) Subcritical in stiff system, $\kappa=5$ and $\lambda=1$.

emerges a finite region of permanently sustained, bounded oscillations of $V$ about $V_{1}$. The crosses show points on the boundary of the region; a change of perturbation parameters $V_{1} / V_{0}$ and/or $\kappa / \kappa_{\mathrm{er}}$, too small to be seen on the figure, changes the response from bounded fluctuations to unstable at the upper and left boundaries and from periodic to constant solutions at the right boundary. (The sustained oscillation region degerates to the vertical line segment $\kappa=\kappa_{\mathrm{cr}}(\equiv \lambda)$ in Fig. 10 for the one state variable friction law; the solid line orbits in Fig. 5 and uppermost curve in Fig. 8 are examples of the oscillations in that case).

For the two state variable friction law, response within the sustained oscillation region involves motions which ultimately become the periodic stable limit cycles, predicted for stiffness $\kappa$ near but below $\kappa_{\mathrm{cr}}$, by the Hopf bifurcation analysis, Fig. $4 \mathrm{~b}$. The response includes also period-doubled bifurcations of those limit cycle motions 


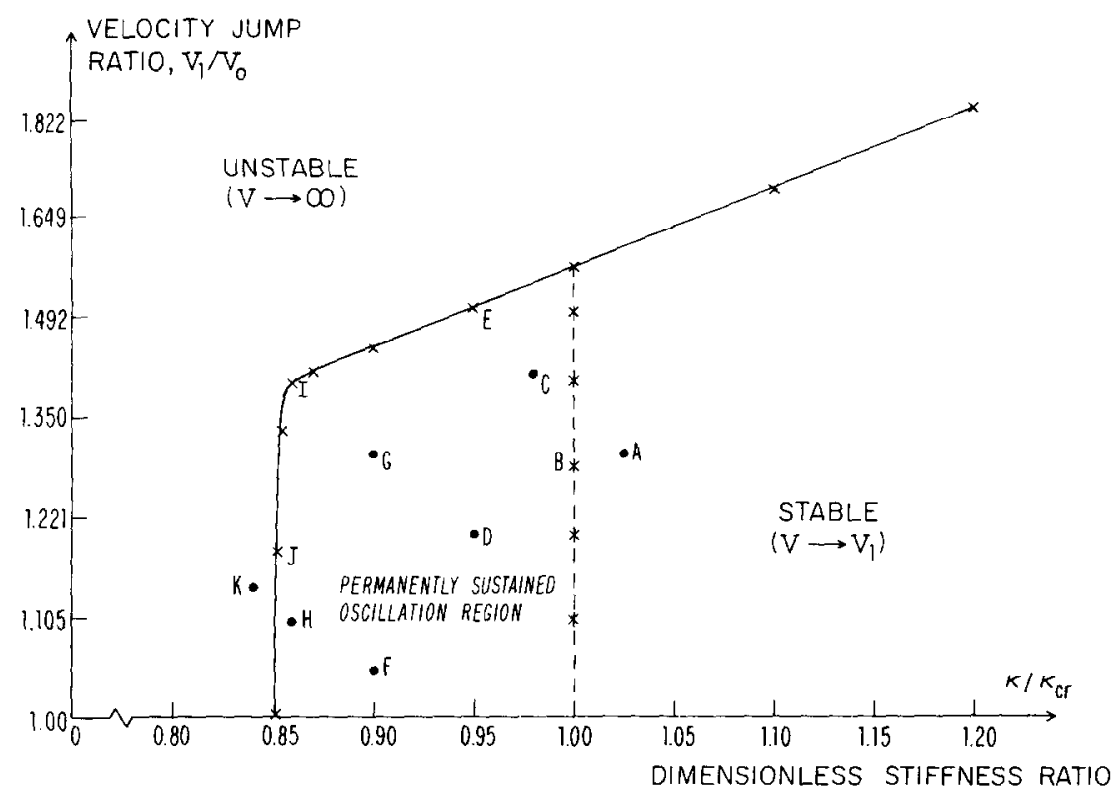

FIG. 13. Stability domains (with approximate determination of boundaries; see text) for system with two state variable friction law, beginning in steady state at speed $V_{0}$ and subject to load point velocity jump to $V_{1}$ as in Fig. 7c. Based on $\beta_{1}=B_{1} / A=1.00, \beta_{2}=B_{2} / A=0.84, L_{1} / L_{2}=0.048$; dimensionless stiffness $\kappa$ is defined consistently with equation $(12 \mathrm{~b})$ and $\kappa_{\mathrm{cr}}=0.876$. Note that region of permanently sustained oscillations (which may be either periodic or chaotic) opens between unstable region ( $V \rightarrow \infty$ in finite time) and stable region (approach to new steady state for speed $V_{1}$ ).

and apparently chaotic (aperiodic) motions, as RUINA (1983) has already shown by imposing a given $V_{1} / V_{0}$ and plotting the numerically integrated response from equations $(6,7)$ for progressively lower values of $\kappa$.

Figure 14 shows our numerically integrated stress versus slip records for each of the perturbations of steady state slip, marked by letters A, B, ., J, K in Fig. 13. The letters are listed in order of decreasing stiffness, and we find that for a given stiffness within the oscillatory region, the same final periodic motion (limit cycle) results no matter what the initial perturbation; compare $D$ with $E, F$ with $G, H$ with $I$. If we examine in succession records $B, C, D$ or $E$, and $F$ or $G$, we find that the amplitude of the limit cycle response increases as $\kappa$ is decreased from $\kappa_{\mathrm{cr}}$, as is expected from the qualitative plot of oscillation amplitude $\varepsilon$ vs $\kappa$ in Fig. 4b. As the stiffness decreases from that for $F$ or $G$ $\left(0.90 \kappa_{\mathrm{cr}}\right)$ to that for $\mathrm{H}$ or $\mathrm{I}\left(0.86 \kappa_{\mathrm{cr}}\right)$ the system passes through a period-doubling bifurcation, as is evident from the altered form of the records. Complicated and possibly chaotic motions result when stiffness is further reduced to a value very close to the left boundary of the sustained oscillation region in Fig. 13; see record $\mathbf{J}$. By analogy with other better studied cases, one may conjecture that the system undergoes a sequence of period-doubling bifurcations as $\kappa$ decreases from $0.86 \kappa_{\mathrm{cr}}$. The left boundary of the sustained oscillation region may possibly coincide with the chaotic limit of an infinite number of such bifurcations, or it may simply coincide with the chaotic-appearing result of a finite number of bifurcations. 


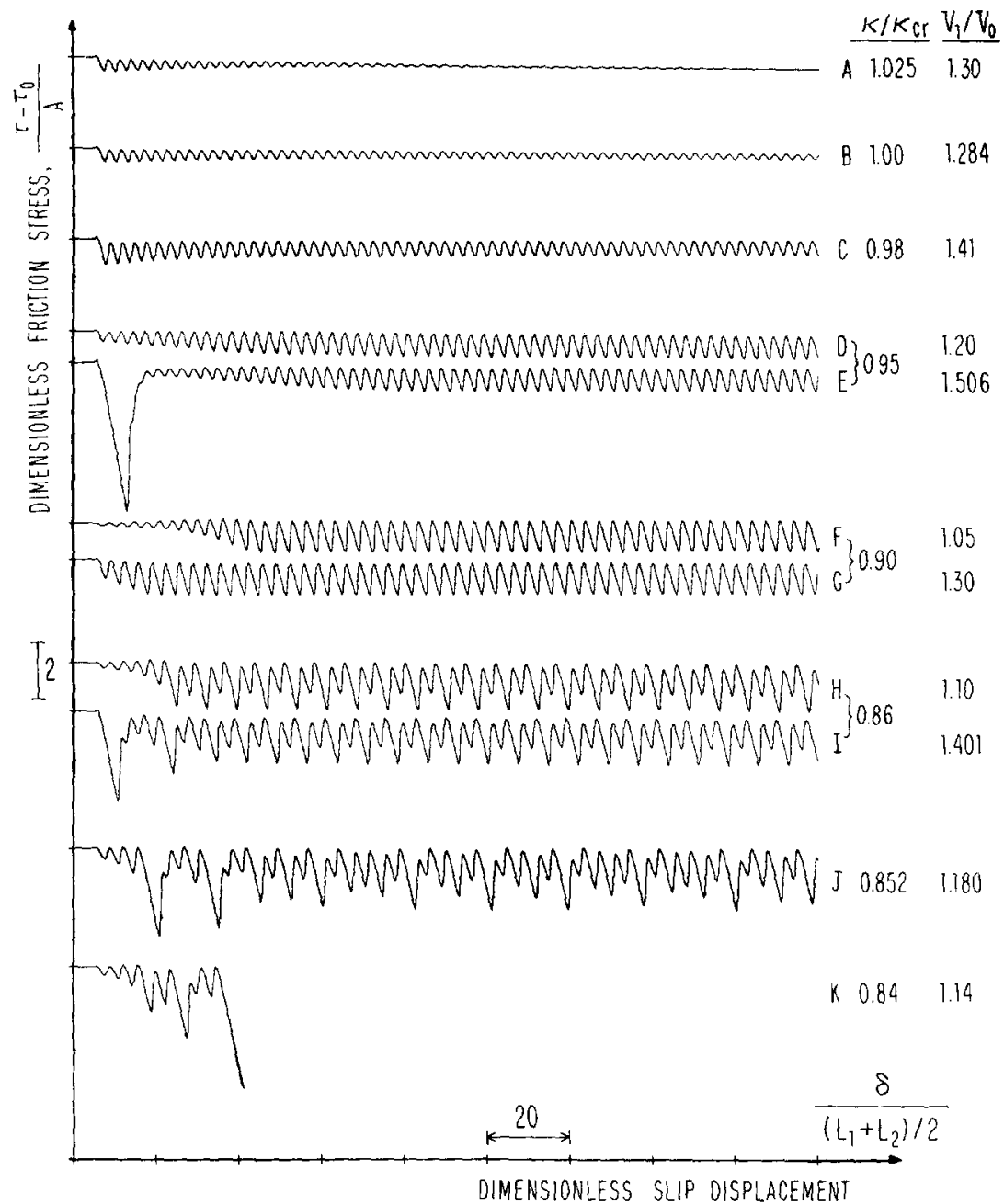

FIG. 14. Some representative stress vs slip records following load point velocity jump perturbations, for values of $V_{1} / V_{0}$ and $\kappa$ within $(\mathrm{B}, \mathrm{C}, \ldots, \mathrm{I}, \mathrm{J})$ or outside $(\mathrm{A}, \mathrm{K})$ the region of permanently sustained oscillations in Fig. 13.

Record A in Fig. 14 is representative of the response to perturbation in the stable region. The system approaches the new steady state $\left(V=V_{1}>V_{0}, \tau=\tau_{1}<\tau_{0}\right)$ with gradually decaying oscillation as shown when $\kappa$ is near to $\kappa_{\mathfrak{c r}}$, but with rapid decay at larger $\kappa$. The route to instability is different at the upper and left boundaries of the sustained oscillation region in Fig. 13. Perturbations at the upper boundary slightly greater than those for $E$ and I cause similar initial response to that shown in Fig. 14, except that the system never recovers from the initial stress drops, during which $V \rightarrow \infty$. Record $\mathrm{K}$ in Fig. 14 shows a typical result just beyond the left boundary; the motion is initially oscillatory with growing amplitude, and ultimately a stress decrease becomes large enough that there is no recovery and $V \rightarrow \infty$. 


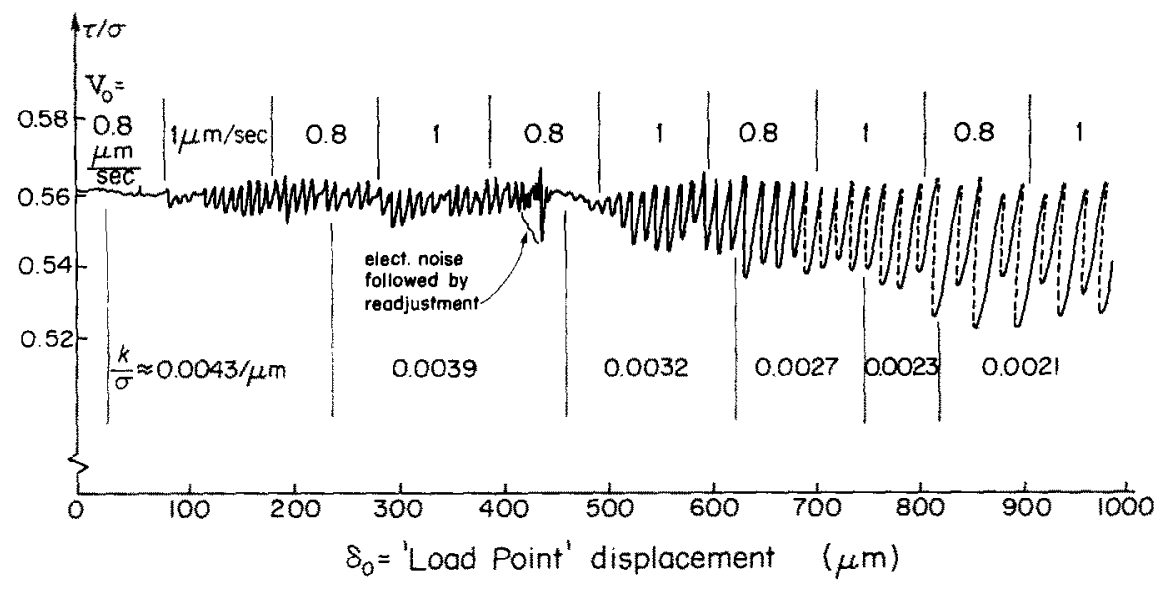

(c)

FIG. 15. Experimental record for polished quartzite at constant normal stress (RUINA, 1980), showing effect of perturbations of load point speed $V_{0}$, stepped between 0.8 and $1.0 \mu \mathrm{m} / \mathrm{sec}$, and of stepped decreases in the spring stiffness $k$, simulated by servocontrol, on generation of oscillations. Apparent decreases of $\delta_{0}$ represented by dashed line segments due to inadequacy of control system during rapid stress drops.

The oscillation amplitudes in Fig. 14 can be compared to the scale division of 2 shown for the dimensionless quantity $\left(\tau-\tau_{0}\right) / A$. Since the initial steady state stress $\tau_{0}$ would typically be of order $60 A$ (e.g. in Figs. $3 \mathrm{c}, \mathrm{d}, \tau_{0} / \sigma \approx 0.55$ to 0.60 and $A / \sigma \approx 0.011$ ), this scale division represents a stress fluctuation of the order of 3 to $4 \%$ from the steady state value. Hence the stress oscillation that we show in Fig. 14 represents a small fraction of the total friction stress, but this small fraction (more precisely, the constitutive relation which governs it) is critical to determining whether slip at constant normal stress is stable or not.

Experimental results by RuINA (1980) for sliding on polished quartzite surfaces, reproduced here as Fig. 15, show some but not all of the features just predicted. Ruina continually stepped the load point speed $V_{0}$ between 0.8 and $1.0 \mu \mathrm{m} / \mathrm{sec}$, and reduced the effective spring stiffness $k$ in steps. This stiffness alteration was simulated electronically through servocontrol, there being no actual change made in any elastic elements of the test apparatus. Sliding at high stiffness $k$ was stable, but oscillations developed as stiffness was reduced to the range shown, in the plot of $\tau / \sigma$ vs load point displacement $\delta_{0}$. They do not seem to be characterizable as simple limit cycle oscillations, perhaps because the system is continually re-excited by electronic noise or perhaps because there was an inadequate wait before a new perturbation was applied. Nevertheless, two features of the experimental response shared by our calculations are: (1) the amplitude of the observed oscillation seems generally to increase with decreases in simulated stiffness, and (2) the oscillations exhibit break-ups into repeated pairs of peaks that appear to be compatible with period-doubling.

The quantity $\delta_{0}$ is shown to decrease (dashed lines) during rapid stress drops at low stiffness. This happens because the servocontrol responds to a feedback signal $\delta_{0}$, interpreted as load point displacement, which is the sum of a displacement measured 
somewhat remote from the slip surface and a constant times the measured force; variation of the constant simulates change of stiffness. The control is intended to make $\mathrm{d} \delta_{0} / \mathrm{d} t$ coincide with the prescribed $V_{0}$, but it cannot do this accurately during very rapid drops in force and, instead, there result the slight decreases in the sum $\delta_{0}$ shown by the dashed lines.

Our prediction of the critical stiffness from equation $(12 b)$ is $\kappa_{\mathrm{cr}}=0.876$, using constitutive parameters cited earlier for the Ruina quartzite data. Since $A / \sigma=0.011$ and $L_{1_{1}}+L_{2}=5.45 \mu \mathrm{m}$ in this case, the corresponding prediction of $k_{\mathrm{cr}} / \sigma$ is $0.0035 / \mu \mathrm{m}$. This may be compared to the value $0.0043 / \mu \mathrm{m}$ at which the stable range is found experimentally to be passed in Fig. 15.

\section{CONClusions ANd Discussion}

Quasi-static slip motion and stability has been analyzed for single degree of freedom elastic systems, Fig. 1, loaded by imposed spring motions. These systems follow rate and state dependent friction laws that incorporate approximately features observed in rock sliding experiments. Most results presented are for the one state variable law of equations (4), Fig. 2; some are for the more elaborate two state variable law of equations (6), Fig. 3. The laws incorporate positive instantaneous and negative long term (steady state) dependence of friction stress on slip speed $\boldsymbol{V}$, with stress changes of both types proportional to $\ln v$.

The critical spring stiffness $k_{\mathrm{cr}}$, from linearized analysis of motions near steady state slip, was found to retain significance for general non-linear motions and their stability. This critical stiffness has the property that within linearized theory, slip in a steady state consistent with uniform imposed load point speed $V_{0}$ is stable if $k>k_{\mathrm{cr}}$, unstable of $k<k_{\mathrm{cr}}$, and shows sinusoidal oscillations of $V$ about $V_{0}$, in response to small perturbations, when $k=k_{\mathrm{cr}}$. The oscillations decay or grow in amplitude when $k$ is slightly increased or decreased, respectively, from $k_{\mathrm{cr}}$. The critical stiffness is given by equations $(12,13)$ for the adopted friction laws and is independent of slip speed for them.

For a system subject to the one state variable friction law, and on which motion at uniform speed $V_{0}$ is imposed at the load point, we have shown (by combination of direct trajectory determination and Liapunov methods, equations (18) to (21) and Fig. 5) that: (i) All possible motions with stiffness $k<k_{\mathrm{er}}$ are unstable in the sense that $V \rightarrow \infty$ in finite time. (ii) When $k=k_{c r}$, all motions which begin sufficiently close to steady state exhibit permanently sustained finite amplitude periodic oscillations of $V$ about $V_{0}$; those which begin further from steady state are unstable. (iii) All motions with stiffness $k>k_{\text {cr }}$ are stable (in that a steady state is approached with $V \rightarrow V_{0}$ ) if the motions begin sufficiently close to steady state; those which begin sufficiently far away are unstable.

Boundaries between the stable and unstable responses mentioned above have been determined for some specific types of finite perturbations of systems that begin in steady state slip. Load point velocity jump (Fig. 7c) and relaxational (Fig. 7d) perturbations are considered; in the latter, motion of the load point is stopped for some time and then resumed at uniform speed. Results show that in systems with $k>k_{\mathrm{cr}}$ the amplitude of perturbation (velocity ratio, relaxation time) necessary to induce unstable 
subsequent motion increases exponentially with $k$ at large $k$ (Figs. 10, 11). Also, the stress versus slip relations that follow such perturbations exhibit sharp peaks of strength followed by a rapid decay either to instability or to the appropriate steady state level, with or without oscillations (Figs. 8, 12).

The possible trajectories in a phase plane, Fig. 6 , of systems following the one state variable friction law and having a stationary load point are determined explicitly, (25). Motions are stable (standard relaxation behavior) or unstable ( $V \rightarrow \infty$ in finite time) according to whether they start below or above a dividing straight line in the phase plane, and it is shown that all motions, whether with stationary or moving load points, are unstable if they reach states above that dividing line.

Insofar as we have determined the motion and stability of a system subject to the two state variable friction law, results are qualitatively similar to those for a system subject to the one variable law, except that considerably more complicated motions are possible for $k$ near to but less than $k_{\mathrm{cr}}$. The first suggestion of this was provided by the Hopf bifurcation analysis (Fig. 4b), through which stable limit cycle oscillatory solutions were shown to exist, in response to motion of the load point at uniform speed, for $k$ in some small neighborhood below $k_{\mathrm{cr}}$. A numerical investigation of the response of a specific two state variable system, beginning in steady state slip, to a sudden jump in load point velocity $\left(V_{0}\right.$ to $\left.V_{1}\right)$ is summarized in Fig. 13 . It shows unstable $(V \rightarrow \infty)$ and stable $\left(V \rightarrow V_{1}\right)$ regions, located similarly (in a $V_{1} / V_{0}$ vs $k / k_{\mathrm{cr}}$ plane) to those for the one state variable analysis, but also shows a region of permanently sustained oscillations of $V$ about $V_{1}$. Representative examples of these oscillations, Fig. 14, were shown to include not only the periodic limit cycle oscillation from the Hopf analysis, but also a period-doubled bifurcation of it, and apparently chaotic bounded oscillations.

We emphasize that the results here follow specifically from quasi-static analysis of single degree of freedom systems slipping in conformity with the friction laws of equations (4) and (6). It remains an open question as to what extent they are representative of response for more complex systems or for systems following other realistic friction laws of the state variable form.

\section{ACKNOWLEDGEMENT}

This work was supported by NSF grants and USGS contracts at Brown, Cornell and Harvard Universities. J.R.R. is grateful for helpful discussion with L. N. Howard and P. C. MARTIN, and A.L.R. for programming assistance from D. BREMNER and comments from J. JENKINS, R. RAND, F. Horowitz and E. GARNICK.

\section{REFERENCES}

Brace, W. F, and BYerLeE, J.D. Brockley, C. A. and Ko, P. L. BYERLEE, J.D.

DIETERICH, J. H.

DiETERICH, J. H.

DiETERiCh, J. H.
J. Geophys. Res. 84, No. B5, 2161-2168.

1979 b J. Geophys. Res. 84, No. B5, 2169-2175.

Science, 153 No. 3739, 990-992.

J. Lubrication Tech. 92, 550-556.

rectonophysics 9, 4/5-486.

Pure Appl. Geophys. 116, 790-806. 
DieTERICh, J. $\mathrm{H}$.

DiETERICH, J. H.

Hassard, B. D., Kazarinoff, N. D. and WAN, Y.H.

HOWARD, L. N.

HigGs, N. G.

JENKIN, F. and EWING, J.A.

JOHNSON, $T$.

Kosloff, D. D. and LiU, H.-P.

MAvKo, G. M.

RabinowiCZ, E.

RABINOWICZ, E.

RICE, J. R.

RICE, J. R. and RUINA, A. L. RUINA, A. L.

RUINA, A. L.

Sampson, J. B., Moran, F., ReEd, P. W. and Muskat, M. STOKER, J. J.

Teufel, L. W.
1980 Solid Earth Geophysics and Geotechnology (edited by S. Nemat-Nasser) Appl. Mech. Div., Vol. 42, Am. Soc. of Mech. Eng., NY, pp. 21-30

1981 Mechanical Behavior of Crustal Rocks (the Handin Volume) (edited by $\mathrm{N}$. L. CARTER, M. Friedman, J. M. Logan and D. W. STEARNS) Geophysical Monograph Series, No. 24, American Geophysical Union, pp. 103-120.

1981

Theory and Applications of Hopf Bifurcation, London Math Soc. Lecture Note Series, Vol. 41, Cambridge University Press, Cambridge.

1979 Nonlinear Oscillations in Biology, (edited by F. C. HopPENSTEADT), Lectures in Appl. Math., Vol. 17, Amer. Math. Soc., Providence, pp. 1-67.

1981

"Mechanical Properties of Ultrafine Quartz, Chlorite and Bentonite in Environments Appropriate to Upper-Crustal Earthquakes," Ph.D. Thesis, Texas A\&M University, August 1981.

1877 Phil. Trans. R. Soc. 167, 508-528.

1981 J. Geophys. Res. 86, No. B7, 6017-6028.

1980 Geophys. Res. Letts. 7, No. 11, 913-916.

1980 EOS, Trans. Am. Geophys. Union 61, No. 46, (abstract) 1120.

1958 Proc. Phys. Soc. 71, part 4, 668-675.

1959 Friction and Wear, Proc. of the Symp. on Friction and Wear, (edited by R. DAvies) Elsevier Publishing Co., pp. 149-164.

1980

Physics of the Earth's Interior (Proc. of the Internat'l School of Physics "Enrico Fermi", course 78, 1979), (edited by A. M. DzIEWONSKI and E. BosCHI) Italian Physical Society, North Holland Publ. Co. 555-649.

1983 Trans. ASME, J. of Appl. Mech. 50, 343-349.

1980 "Friction Laws and Instabilities: A Quasistatic Analysis of some Dry Frictional Behavior," Ph.D. Thesis, Brown University, Providence, RI.

1983 J. Geophys. Res. 88, No. B12, 10359-10370.

1943 J. Appl. Phys. 14, 689-700.

1950 Nonlinear Vibrations in Mechanical and Electrical Systems, Interscience Publishers, John Wiley \& Sons, Inc.

1981 "Frictional Instabilitites in Rock: Effect of Stiffness, Normal Stress, Sliding Velocity, and Rock Type," presented at the 18th Annual Meeting of Soc. for Eng. Sci., Brown University, Providence, RI, September 2-4, 1981. 